\title{
Inflammation promotes tumor aggression by stimulating stromal cell- dependent collagen crosslinking and stromal stiffening
}

Ori Maller ${ }^{1^{*}}$, Allison P. Drain ${ }^{{ }^{*}}$, Alexander S. Barrett ${ }^{2^{*}}$, Signe Borgquist ${ }^{3,4}$, Brian Ruffell ${ }^{5}$, Pham T Thanh $^{2}$, Tina Gruosso ${ }^{6}$, Hellen Kuasne ${ }^{6}$, Johnathon N. Lakins ${ }^{1}$, Irene Acerbi ${ }^{1}$, J. Matthew Barnes ${ }^{1}$, Travis Nemkov ${ }^{2}$, Aastha Chauhan ${ }^{7}$, Jessica Gruenberg ${ }^{7}$, Aqsa Nasir ${ }^{7}$, Olof Bjarnadottir ${ }^{3}$, Zena Werb ${ }^{8,9}$, Peter Kabos ${ }^{10}$, E. Shelley Hwang ${ }^{12}$, Morag Park ${ }^{6}$, Lisa M. Coussens ${ }^{5}$, Andrew C. Nelson ${ }^{7}$, Kirk C. Hansen ${ }^{2,14}$ and Valerie M. Weaver ${ }^{9,13,14,15}$

${ }^{1}$ Department of Surgery, Center for Bioengineering and Tissue Regeneration, University of California, San Francisco, California, USA

${ }^{2}$ Department of Biochemistry and Molecular Genetics, University of Colorado Denver - Anschutz Medical Campus, Aurora, CO, USA

${ }^{3}$ Division of Oncology and Pathology, Department of Clinical Sciences, Lund, Lund University ${ }^{4}$ Clinical Trial Unit, Clinical Studies Sweden, Forum South, Skåne University Hospital, Lund, Sweden

${ }^{5}$ Cell, Developmental \& Cancer Biology, Oregon Health \& Science University; Knight Cancer Institute, Oregon Health \& Science University, Portland, Oregon, USA

${ }^{6}$ Goodman Cancer Research Centre, McGill University, Montreal, QC, Canada; Department of Biochemistry, McGill University, Montreal, QC, Canada; Department of Oncology, McGill University, Montreal, QC, Canada

${ }^{7}$ Department of Laboratory Medicine and Pathology, University of Minnesota, Minneapolis, MN, USA

${ }^{8}$ Department of Anatomy and Biomedical Sciences Program, University of California, San Francisco CA, USA

${ }^{9}$ UCSF Helen Diller Comprehensive Cancer Center, University of California, San Francisco, San Francisco, CA, USA

${ }^{10}$ Department of Medicine, Division of Medical Oncology, University of Colorado Anschutz

Medical Campus, Aurora, Colorado, USA

${ }^{11}$ Department of Pathology, University of California, San Francisco, CA, USA

${ }^{12}$ Department of Surgery, Duke University Medical Center, Durham, NC, USA

${ }^{13}$ Departments of Bioengineering and Therapeutic Sciences, and Radiation Oncology, Eli and Edythe Broad Center of Regeneration Medicine and Stem Cell Research

*These authors contributed equally to this work

${ }^{14}$ These authors jointly supervised this work

The authors have no conflicts of interest to declare.

\author{
${ }^{15}$ Corresponding Author: \\ Valerie M. Weaver \\ Center for Bioengineering and Tissue Regeneration \\ Department of Surgery \\ University of California, San Francisco \\ Telephone: (415) 476-3826 \\ Email: valerie.weaver@ucsf.edu
}




\section{Abstract}

2 Collagen deposition and stromal stiffening accompany malignancy, compromise treatment, and

3 promote tumor aggression. Clarifying the molecular nature of and the factors that regulate

4 extracellular matrix stiffening in tumors should identify biomarkers to stratify patients for therapy

5 and therapeutic interventions to improve outcome. We profiled lysyl hydroxylase- and lysyl

6 oxidase-mediated collagen crosslinks and quantified the greatest abundance of total and complex

7 collagen crosslinks in more aggressive human breast cancer subtypes with the stiffest stroma.

8 These tissues also harbored the highest number of tumor-associated macrophages (TAM), whose

9 therapeutic ablation not only reduced metastasis, but also concomitantly decreased accumulation

10 of collagen crosslinks and stromal stiffening. Epithelial-targeted expression of the crosslinking

11 enzyme lysyl oxidase had no impact on collagen crosslinking in PyMT mammary tumors, whereas

12 stromal cell targeting did. Consistently, stromal cells in microdissected human tumors expressed

13 the highest level of collagen crosslinking enzymes. Immunohistochemical analysis of a cohort of

14 breast cancer patient biopsies revealed that stromal expression of lysyl hydroxylase two, an

15 enzyme that induces hydroxylysine aldehyde-derived collagen crosslinks and stromal stiffening

16 correlated significantly disease specific mortality. The findings link tissue inflammation, stromal

17 cell-mediated collagen crosslinking and stiffening to tumor aggression and identify lysyl

18 hydroxylase two as a novel stromal biomarker.

\section{Significance}

21 We show infiltrating macrophages induce stromal fibroblast, and not epithelial, expression of

22 collagen crosslinking enzymes that drive tumor stiffening. Stromal enzyme LH2 is significantly

23 upregulated in breast cancer patients with the stiffest stroma, the most trivalent HLCCs and the

24 worst prognosis, underscoring its potential as a biomarker and therapeutic target. 


\section{Introduction}

Pathological accumulation of extracellular matrix (ECM) accompanies the formation of all solid tumors(1-3). The tumor ECM is composed primarily of interstitial collagen that is progressively reorganized and stiffened $(2,4)$. The collagenous fibrotic tumor ECM compromises treatment and is linked to poor patient prognosis(5-8). Tumor biopsy analysis showed that a

31 thick fibrous collagenous ECM associates with less differentiated tumors and that this

32 phenotype predicts poor patient survival, emphasizing the relevance of collagen

33 architecture $(1,4,9)$. Patients with pancreatic ductal adenocarcinomas (PDACs) that are

34 surrounded by stiff, thick fibrous collagens have a shorter survival, and invasive breast

35 carcinomas with the stiffest ECM stroma at their invasive front are the most aggressive $(1,2)$.

36 These observations suggest that stromal stiffness reflects collagen organization may be an

37 important prognostic variable. Consistently, preclinical studies using organotypic cultures and

38 rodent models provide plausible evidence for a causal relationship between collagen organization, stromal stiffness, tumor cell invasion in culture, and metastasis in vivo(10-14).

40 These findings underscore the clinical relevance of collagen architecture and stiffness to

41 malignancy, and emphasize the need to clarify the molecular nature of the collagenous ECM so

42 that new biomarkers can be identified and anti-cancer therapeutics may be

43 developed $(1,2,15,16)$.

Interstitial type I fibrillar collagen is the ECM component that contributes most significantly to the tensile strength of tissue(17). The tensile strength of interstitial collagen depends upon the activities of two major families of enzymes: the lysyl hydroxylases (LH; gene

47 name procollagen-lysine, 2-oxoglutarate 5-dioxygenase or PLOD) and the lysyl oxidases (LOX),

48 which regulate fibrillogenesis of newly synthesized collagen molecules through intermolecular

49 covalent crosslinking(18-21). Fibrotic human tumors express high levels of LOX and LH

50 enzymes(22). Tumor grade and overall patient survival associate with total tissue LOX and

51 PLOD2 mRNA(21,23-25). Pharmacological or antibody-mediated inhibition of LOX in MMTV- 
52 Her2/Neu mice or genetic reduction of PLOD2 in subcutaneously-injected lung tumor epithelial

53 cells reduce tissue fibrosis, stromal stiffening and collagen crosslinking and concomitantly

54 decrease tumor incidence and aggression(12,23). Moreover, elevating LOX or LH2-mediated

55 collagen crosslinking enhances fibrosis and stromal stiffness and promotes malignant

56 transformation and tumor aggression in lung and mammary xenografted tumors(12,23). These

57 observations suggest that the direct targeting of specific collagen crosslinking enzymes has

58 clinical merit for the treatment of cancer. However, given caveats with recent clinical trials

59 targeting ECM modifiers including suboptimal activity of inhibitory treatments and the risk of off-

60 target effects, strategies designed to interfere with the induction and activation of these

61 crosslinking enzymes offer an attractive alternative(26). Towards this goal, the identification and

62 causal implication of additional factors that regulate the levels and/or activity of collagen

63 crosslinking enzymes has the potential to identify new predictive biomarkers and alternative

64 anti-tumor treatment targets.

Pre-neoplastic lesions are inflamed, and pathological fibrosis correlates with

inflammation $(27,28)$. Chronic inflammation and experimental manipulations that promote

67 inflammation in rodent models induce fibrosis by secreting factors such as metalloproteinases

68 and TGF $\beta(14,28-31)$. Furthermore, fibrotic tumors are frequently inflamed, and this inflammation

69 promotes tumor aggression, whereas either inhibiting inflammation or decreasing macrophage

70 infiltration reduce tumor metastasis and enhance anti-tumor treatment(2,27,32-35).

71 Nevertheless, it remains unclear if inflammation promotes tumor progression and aggression by

72 inducing stromal stiffening, and if so, whether this is regulated via epithelial and/or stromal

73 fibroblast-mediated collagen remodeling and crosslinking.

\section{$74 \quad$ Results}

75 XAAA profiling identifies increased levels of collagen crosslinks and stromal stiffness as

76 indicators of breast tumor aggression 
To clarify the role of collagen crosslinking in tumor fibrosis we developed a crosslinked amino acid analysis (XAAA) method that enabled the characterization and quantification of specific collagen crosslinks in tissues across a wide range of collagen levels. We utilized solid phase extraction (SPE) enrichment followed by high $\mathrm{pH}$ amide hydrophilic chromatography (HILIC) coupled to a benchtop orbitrap (QExactive) mass spectrometer for these measurements. The validated method detected all known LOX-generated crosslinks including divalent (lysinonorleucine, dihydroxy lysinonorleucine), trivalent (pyridinoline and deoxypyridinoline) and tetravalent (desmosine and isodesmosine) crosslinked amino acids linearly over four orders of magnitude, with calculated limits of quantification (LLOQ) in the femtomolar range (Suppl. Fig. 1; Suppl. Table 1)(36). The technique revealed a positive correlation between collagen crosslinking and abundance in excised human clinical specimens with very low to very high collagen concentrations and varying mechanical properties (Suppl. Fig 2). The method also identified a subset of hydroxylysine aldehyde $\left(\mathrm{Hy}^{\mid \mathrm{ld}}\right)$-derived collagen crosslinks (HLCCs) crucial for the mechanical strength of tissue $(17,37)$. between 22 and 58) and human tumor biopsies representing early stage (stage 1-2) invasive breast cancers (IBC) excised from mastectomy specimens. Molecular subtyping that subdivides

94 human breast tumors stratified by estrogen receptor (ER+) and human epidermal growth factor

95 receptor two (HER2+) status and ER/PR/HER2-negative (triple negative; TN) is a key

96 determinant used to direct the treatment of breast cancer patients. Accordingly, we chose

97 human breast tumor biopsies that represented $\mathrm{ER}+(\mathrm{N}=8$; age between 42 and 71); HER2+

98 ( $N=6$; age between 40 and 76) and TN ( $N=6$; age between 50 and 71). H\&E stained tissue

99 sections confirmed the presence of normal glandular structures in the normal controls and

100 invasive breast cancer in the tumor specimens (Fig. 1a; top panels). Polarized light imaging of

101 Picrosirius-stained (PS) tissue revealed that the normal breast tissue stroma had very little

102 fibrillar collagen, whereas stromal tissue in all patients with IBCs contained abundant fibrillar 
collagen (Fig. 1a; middle panels) that second harmonic generation (SHG) imaging indicated was thicker and more linearized (Fig. 1a; bottom panels). Polarized light microscopy and twophoton imaging further revealed that the level of fibrosis in the tissue was higher in HER2+ as compared to the ER+ breast tumors, and was further increased in the TN tumors, consistent with our previous report that TN tumors contain a high density of aligned collagen fibers (Fig. 1a; middle and bottom panels)(2). AFM microindentation revealed a significant increase in the elastic modulus of the stroma associated with the invasive front of all the IBC tissues (Fig. 1b) $(2,4,12)$. XAAA analysis revealed a significant increase in total collagen crosslinking in all the

111 IBCs (Fig. 1c, Suppl. Fig. 5). These findings are consistent with an association between collagen crosslinks, tissue fibrosis and stromal stiffness, as has been previously documented in experimental murine models of mammary cancer(12). Interestingly, when we subdivided the

114 IBC collagen crosslinking analysis into breast tumor subtype the most significant increase in total collagen crosslinks was calculated to be in the TN breast tumors (Fig. 1i). Furthermore, biochemical quantification of total tissue collagen $1 \mathrm{~A} 1$ or $1 \mathrm{~A} 2$ did not account for the higher total number of collagen crosslinks in the TN breast tumor tissue (Fig. 1k). Instead, molecular characterization of the isolated tissue collagen revealed that the TN tumors had a distinctive crosslink profile due to a strong preference for a combination of DHLNL, Pyr, and d-Pyr crosslinks (Fig. 1d-j). Subtype analysis further revealed that the level of hydroxylysine

121 aldehyde-derived collagen crosslinks (HLCCs) in the TN subtype correlated significantly and

122 positively with the stiffness of the stroma at the invasive front of the tumor tissue (Fig. 1I). These 123 findings highlight the importance of HLCC collagen crosslinking in breast cancer aggression.

\section{Increased collagen crosslinking correlates with high expression of stromal LOX and}

\section{PLOD2 in aggressive tumor subtypes}

To determine if the observed increase in collagen crosslinking and stromal stiffness in

127 TN tumors was related to the expression levels of enzymes implicated in regulating collagen 128 crosslinking, we analyzed publicly available human breast cancer gene expression array data (n 
$129=1904)$ for the genes coding these enzymes and examined their correlation to breast cancer

130 subtype. Bioinformatics analyses revealed a significant increase in the major collagen

131 crosslinking enzyme LOX, but not lysyl oxidase like two (LOXL2), in the more aggressive HER2

132 and TN tumor subtypes and indicated that LOX levels were particularly high in TN tumors (Fig.

133 2a-b). The arrays also showed bulk gene expression of PLOD2, the major regulator of HLCC

134 accumulation, to be highly upregulated in aggressive human TN breast cancers (Fig. 2c).

135 To gain insight into the cellular sources of LOX and LH2 in human tumors, we used laser

136 capture microdissection to isolate regions of tumor epithelium and stroma to identify the origins

137 of LOX and LH2 in invasive human breast cancers $(38,39)$. Gene expression analysis of stromal

138 and epithelial compartments revealed that the stromal cells in the tumor tissue expressed

139 significantly more LOX and PLOD2 than the associated tumor epithelium. The data further

140 indicated that this relationship was more evident in the breast tissue from women with ER-/PR-

141 breast cancer that is frequently the more aggressive tumor subclass (Fig. $\mathbf{2} \mathbf{d}-\mathbf{g}$ ).

142 The findings implicate, but do not definitively demonstrate a role for LOX and PLOD2 in

143 generating the increased level and greater complexity of collagen crosslinking we quantified in

144 the more aggressive human breast tumors. Nevertheless, the combination of these gene

145 expression data with our findings showing increased collagen crosslink abundance provide

146 compelling evidence to suggest that increased expression of LOX and PLOD2, particularly from

147 stromal cells, likely contribute to elevated levels of collagen crosslinks and HLCCs in TN tumors.

148 Moreover, given that TN tumors are the most aggressive and lethal breast cancer, these data

149 link human breast tumor aggression to increased levels of total and complex collagen crosslinks

150 and higher stromal stiffness. Accordingly, the findings implicate the collagen crosslinking

151 enzymes LOX and LH2, and by extension factors that regulate their expression, in breast

152 cancer aggression.

153 Stromal - and not epithelial - crosslinking enzymes regulate tissue fibrosis and

154 collagen crosslinking in vivo 
Prior studies demonstrated that both cancer cell lines and stromal fibroblasts express LOX and LH2 to induce tissue stiffening and fibrosis implying they also drove collagen crosslinking. Seminal articles identified hypoxia-induced HIF1a as a key regulator of tumor epithelial LOX and LH2 expression and suggested epithelial secretion of these enzymes drives collagen remodeling, crosslinking and stiffening that foster tumor cell dissemination and primes the premetastatic niche to facilitate metastatic colonization $(22,24,25)$. Our analysis of breast cancer clinical specimens showed that the stromal cells in the breast tissue express higher levels of

162 LOX and PLOD2 as compared to the breast tumor epithelium. Moreover, our prior studies

163 showed LOX is expressed in stromal fibroblasts in a transgenic mouse model of ErbB2-induced

164 mammary tumor malignancy. Furthermore, we demonstrated that fibroblasts expressing LOX

165 injected into a cleared mammary fat pad not only induced ECM remodeling and stiffening but also potentiated the growth and malignant progression of pre-malignant tumor cells injected into the modified glands (12). Thus, the relative contribution of tumor- and stromal- derived collagen crosslinking enzymes to tumor fibrosis, ECM remodeling and collagen crosslinking remains unclear; particularly in the context of spontaneous tumors and patient tumors in which the ECM evolves concurrently with tumor progression.

172 induce tissue fibrosis and stromal stiffening in mammary tissue, we created a genetically

173 engineered mouse model (GEMM) in which we targeted and controlled luminal epithelial-

174 specific expression of mouse LOX using the MMTV-rtTA promoter (Epithelial LOX

175 overexpression [OX]). (Fig. 3a, Suppl. Fig. 6a-b). We crossed these mice into the PyMT

176 spontaneous mammary tumor model to enhanced LOX expression in the mammary tumor

177 epithelium (PyMT epithelial LOX OX) and assayed their stromal phenotype as compared to the

178 mammary glands from age-matched PyMT control mice (PyMT). Again, despite confirming

179 ectopic LOX expression and elevated levels of cleaved LOX protein in the mammary tumor

180 epithelial compartment (Suppl. Fig 6c), we were not able to detect any increase in the levels, 
nor any altered organization of the mammary gland interstitial collagen (Fig 3b-c). Moreover, XAAA crosslinking analysis revealed that the level of collagen crosslinks between the PyMT control and PyMT epithelial LOX OX mammary gland stroma were indistinguishable (Fig. 3d-i). Nevertheless and importantly, we could easily and consistently detect a significant increase in fibrillar collagens, collagen crosslinks and stromal stiffness in the PyMT mammary tumors as compared to age-matched FVB mammary glands lacking tumors (Fig. 4c,f-k). These studies both validate the sensitivity of our crosslinking assay and imply that tumor epithelial LOX is not the primary driver of collagen crosslinking and stiffening in endogenous mammary tumors. We and others have implicated stromal fibroblast LOX as a key promoter of epithelial tumor progression and aggression $(12,14,40)$. To directly test the functionality of the ectopicallyexpressed LOX and the relevance of fibroblast-specific expression of LOX, we next created mouse cohorts of PyMT GEMMs in which we restricted ectopic LOX expression to the stromal population using the Col1a1-tTA promoter (MMTV-PyMT+/-; Col1a1-tTA+/-;TetO_mLox+/-; herein denoted PyMT LOX OX) (Fig. 4a, Suppl. Fig. 7b). To begin with, induction of LOX in the stromal cells markedly enhanced the amount of fibrillar collagen in the MMTV-PyMT LOX OX mammary glands, as revealed by quantification of polarized images of Picrosirius Red stained tissue (Fig. 4b, second row images; quantified in c). We also detected more and thicker linearized interstitial collagen when LOX was increased in the stromal cells by two-photon second harmonic generation imaging (Fig. 4b, third row panels). In addition, immunostaining revealed more phosphorylated tyrosine 397 focal adhesion kinase protein ( $\left.{ }^{\mathrm{PY} 397} \mathrm{FAK}\right)$ in the mammary epithelium of the glands in which stromal LOX was elevated (Fig. 4b, bottom

202 panels), likely reflecting the increase in elasticity that we measured in the tissue stroma using 203 atomic force microscopy (AFM) indentation(12) (Fig. 4d-e \& Suppl. Fig. 9b). Consistently, we 204 measured higher levels of total collagen crosslinks in the PyMT LOX OX mice as compared to 205 the levels quantified in the MMTV-PyMT control glands (Fig. 4f). Furthermore, the most 206 significant increases that we quantified in the PyMT LOX OX glands were dihydroxy 
207 lysinonorleucine (DHLNL) and pyridinoline (Pyr) crosslinks (Fig. 4g-k), which are the crosslinks

208 generated through the HLCC pathway that promote mechanical stability and strength in skeletal

209 tissue $(37,41)$. Of note, we did not detect any increase in DHLNL or Pyr in the mammary glands

210 in which ectopic LOX expression was elevated in the mammary epithelium using the MMTV

211 promoter (Fig. 3g-h). These findings imply that stromal cells are the primary regulators of

212 interstitial collagen crosslinking and stromal stiffening in mammary tumors.

213 Tissue inflammation regulates tissue fibrosis, collagen crosslinking and stromal

214 stiffening

215 Cancer progression is accompanied by tissue inflammation and the most aggressive

216 human breast tumors with the stiffest invasive stroma harbor the highest number of

217 macrophages(2,35). Consistently, decreasing the number of tumor macrophages, either through

218 genetic ablation of macrophage colony stimulating factor (CSF-1) or via pharmacological and

219 inhibitory antibody treatment with anti-CSF1 antibody, reduces lung metastasis in the PyMT

220 mouse model of mammary cancer(33,34,42,43). Interestingly, macrophage ablation was more

221 effective at preventing lung metastasis in the PyMT mammary tumor model when the treatment

222 was initiated early, prior to malignant transformation and coincident with the onset of tissue

223 fibrosis(33). These findings raise the intriguing possibility that macrophage ablation may

224 regulate tumor aggression, at least in part, by promoting collagen remodeling and inducing ECM

225 crosslinking and stromal stiffening (Fig. 3 \& 4).

226 To assess the possibility that there is a causal association between macrophage-

227 mediated tissue inflammation, tumor fibrosis and ECM stiffening and mammary tumor

228 aggression, PyMT mice were treated with anti-CSF-1 antibody or a non-specific lgG control

229 antibody commencing at four weeks of age, prior to the onset of ductal hyperplasia(44). Mouse

230 cohorts (6/treatment group/time point) were sacrificed at eight and eleven weeks of age.

231 Immunostaining confirmed efficient reduction of mammary tumor tissue macrophages at eight

232 weeks of age, as evidenced by significantly reduced F4-80 immunostaining that was also 
233 evident in the eleven week old treated tissue (Fig. 5a top panel, Suppl. Fig. 8a). The excised

234 lungs from the eleven week mice confirmed reduced frequency of lung metastasis in the anti-

235 CSF-1 antibody-treated group (Fig. 5b), consistent with our prior work documenting a significant

236 inhibition of lung metastasis in fourteen week old mice when anti-CSF-1 treatment was initiated

237 at four weeks(33). The mammary glands from the eight and eleven week old mice were excised

238 and analyzed for fibrosis and biomechanical properties (Fig. 5a,c-i). Despite confirming an

239 equivalent number of fibroblasts in the treated and nontreated groups, polarized images of PS

240 stained tissue revealed lower levels of total fibrillar collagen in the stroma of the eight week anti-

241 CSF1 antibody treated group (Fig. 5a, third row panel, Suppl. Fig. 10b-c). AFM

242 microindentation additionally demonstrated that the tissue stroma from both the eight week old

243 (Fig. 5d), and the eleven week old (not shown) mammary glands was softer, likely accounting

244 for the reduced integrin mechanosignaling detected in the CSF1-antibody treated tissue, as

245 revealed by less intense staining for ${ }^{\mathrm{pY} 397} \mathrm{FAK}$ (Fig. 5a, second row panel). Consistently, we

246 also observed reduced levels of Lox mRNA in eight week, anti-CSF1 treated mice by in situ

247 hybridization (Fig. 5a, bottom panel, Fig. 5e). In agreement with prior studies, nearly all the

248 detected Lox mRNA was restricted to stromal cells (Fig 5a, bottom panel)(14). These findings

249 suggest that reducing the level of tumor-associated macrophages (TAMs) not only prevents

250 lung metastasis but also concomitantly reduces tissue fibrosis and stiffening, likely by

251 preventing stromal cell activation.

TAMs secrete abundant TGF $\beta$ that stimulates fibroblast differentiation to a myofibroblast

253 phenotype and induces expression of collagen crosslinking enzymes including LOX(14). PCR

254 analysis of flow activated cell sorted (FACS) cells from the transformed mammary glands of

255 PyMT mice (11 weeks) confirmed that the TAMs expressed by far the highest levels of TGF $\beta$,

256 as compared to neoplastic epithelium and the cancer-associated fibroblasts (Fig. $\mathbf{5 j}$ ). These

257 findings suggest that the tumor infiltrating macrophages could promote fibrosis and stromal 
stiffening through secreted TGF $\beta$; a finding supported by a strong stromal pSMAD2 (SMAD2 pS465/467) in the IgG control antibody-treated PyMT mammary glands (Fig. 5k-I). To examine whether TAM recruitment could regulate collagen crosslinking in human tumors we queried a publicly available human breast tumor gene expression data set for TAM markers and collagen modifying enzymes. Consistent with a role for TAMs in stimulating expression of collagen modifying enzymes, gene expression of the TAM marker CD163 positively correlated with both LOX and PLOD2 but not LOXL2 in human breast tumors $(n=1904)$ (Fig. 5m-o). Moreover, costaining of resected human breast tumors with the macrophage/monocyte marker CD68 and the downstream TGF $\beta$ signaling molecule pSMAD2 Ser465/467 revealed a significant positive correlation between pSMAD2 Ser465/467 and infiltrating tumor macrophages at the invasive

268 front of human breast tumors (Fig. 5p-q). Furthermore, FACS analysis established a significant correlation between the infiltrating tumor associated macrophages, as demonstrated by CD14 ${ }^{\text {hi CD11 }} \mathrm{b}^{+} \mathrm{HLA}-\mathrm{DR}^{+}$cell surface markers normalized to total CD45 infiltrating cells, and the

271 elastic modulus of the invasive front of human invasive breast cancers (Fig. 5r). These findings

272 suggest that tumor inflammation and macrophage secreted factors such as TGF $\beta$ could

273 promote tissue fibrosis by enhancing fibroblast expression of the collagen crosslinking enzymes

274 LOX and PLOD2, but not LOXL2, to induce collagen crosslinking and stromal stiffening.

275 LH2 inhibition reduces lung metastasis and stromal LH2 predicts poor prognosis in 276 breast cancer patients

277 Using the data set generated from the micro-dissected tumors, we next explored the clinical 278 relevance of epithelial vs. stromal LOX and PLOD2 expression by assessing their relative 279 contribution to overall survival in breast cancer patients. Surprisingly, neither stromal cell nor 280 epithelial LOX predicted overall patient survival in this cohort (Fig. 6a-b). However, the findings 281 clearly showed that overexpression of stromal PLOD2, but not epithelial PLOD2, significantly 282 correlated with poor breast cancer patient prognosis (Fig. 6c-d). The data provide further 
evidence that HLCCs, formed primarily through the activities of collagen crosslinking enzymes expressed by stromal cells, promote breast tumor aggression that contributes to poorer overall survival. The findings also highlight the importance of the collagen crosslinking profile and its potential impact on stromal stiffness in tumor aggression.

LH2 (the protein encoded by PLOD2) is a key enzyme that regulates the level of HLCC crosslinking of fibrillar collagen and consequently contributes substantially to the tensile properties of the tissue stroma $(19,23,45)$. We quantified the highest level of HLCC crosslinking and the stiffest invasive front in the breast tissue from women with the most aggressive breast cancers (Fig. 1j). Consistently, when we analyzed the gene expression data from a published cohort of breast cancer patients we observed that ER-/HER2- $(T N ; n=133)$ breast cancers express the highest level of the PLOD2 gene transcript as compared to the levels expressed in HER2+ $(n=73)$ and ER+/HER2- (luminal, n=314) breast tumors (Suppl. Fig. 11a)(46). Further analysis showed that high PLOD2 expression in breast cancer patients with HER2+ and TN tumors significantly predicts reduced distant metastasis-free survival (DMFS), as well as increased risk of relapse in TN tumors (Suppl. Fig. 11b-d)(46,47). These findings implicate PLOD2/LH2 as a key regulator of breast tumor aggression through its ability to induce the HLCC crosslinking of fibrillar collagens that enhance the stiffness of the tumor stroma. aggression, we systemically treated a cohort of PyMT mice with the LH2 inhibitor minoxidil or vehicle (PBS) from five weeks of age until sacrifice at either 11-12 weeks or 13-14 weeks of age

(Fig. 6e). Consistent with its predicted role in enhancing stromal stiffness through modifying the

304 nature of collagen crosslinks, AFM microindentation revealed that the phenotypically-similar

305 collagen-rich ECM adjacent to tumors from the minoxidil treated mice (see polarized imaging of

306 picrosirius red stained tissue) was significantly softer when compared to the vehicle-treated

307 age/stage-matched PyMT mice (Fig. 6f-h). Furthermore, inhibiting LH2 also significantly

308 decreased lung metastasis, causally linking HLCC collagen crosslinking to stiffness-mediated 
breast tumor aggression (Fig. 6i). Our gene expression data from the epithelial and stromal microdissection cohort indicated that stromal PLOD2, but not epithelial PLOD2, strongly predicted poor survival in breast cancer patients. Therefore, we next sought to definitively assess the relative contribution of stromal versus neoplastic epithelial LH2 protein expression to human breast tumor aggression using a large tissue array of annotated human breast tumor biopsies. To accomplish this, we first developed an LH2 immunostaining protocol (IHC) and then we analyzed neoplastic epithelial and stromal expression of the LH2 enzyme in tissue biopsies from a large cohort of histopathologically classified breast cancer patients $(N=505)$ with

317 accompanying clinical information and follow up data (Suppl. Table 2 and Suppl. Table 3). LH2

318 IHC staining in the epithelium and stromal cells was scored as low, intermediate and high and

319 the relationship between epithelial versus stromal expression and breast cancer patient

320 outcome was calculated (Fig. 6j; Suppl Fig. 12a). IHC scoring analysis revealed that only a

321 modest number of patients with poorly differentiated breast tumors expressed moderate to high

322 levels of epithelial LH2 (Suppl. Fig. 12b); a finding that accords with prior links between tumor

323 size, hypoxia and LH2-dependent tumor aggression(23,24). More strikingly however, we

324 observed that a disproportionately high number of breast cancer patients with moderately and

325 poorly differentiated breast tumors had high stromal cell LH2 (indicated by an H score of above

326 230; Fig. 6k). These data and our observation that even well differentiated breast tumors

327 express intermediate levels of stromal cell LH2 ( $\mathrm{H}$ score above 120 and equal or less than 230)

328 imply that stromal cell $\mathrm{LH} 2$, rather than epithelial $\mathrm{LH} 2$, may be a more robust indicator of breast

329 tumor aggression. Consistently, stratification of breast tumor patient analysis into ER+/HER2-

$330(\mathrm{~N}=296) ; \mathrm{HER} 2+(\mathrm{N}=36)$ and $\mathrm{TN}(\mathrm{N}=32)$ showed an enrichment for intermediate and high

331 stromal LH2 H-score in the more aggressive cancer subtypes (Suppl. Table 2 and Suppl.

332 Table 3). Furthermore, we uncovered a significant correlation between high stromal cell LH2 H-

333 score and shorter breast cancer patient-specific survival when adjusted for age at diagnosis

334 (Fig. 6I). We also established a significant association between stromal cell but not epithelial 
335 LH2 levels and survival in lymph node positive breast cancer patients but not lymph node

336 negative breast cancers (Fig. 6m-n; Suppl Fig. 12d-e). These clinical data identify stromal cell

337 LH2 as a novel biomarker with potential to predict metastatic disease and poor patient survival

338 among breast cancers overall, as well as within the highest risk TN breast cancer subtype.

Discussion:

We identified infiltrating macrophages as key regulators of stromal cell-mediated

342 collagen crosslinking, stromal stiffening and tumor metastasis. Our clinical findings corroborated

343 our experimental results, revealing significant associations between macrophages and collagen

344 crosslinking enzymes providing evidence for a critical impact of inflammatory stromal cell-

345 mediated collagen crosslinking and stromal stiffening in tumor aggression and patient outcome.

346 Diverse subsets of myeloid cells account for adverse patient outcomes because they

347 differentially promote angiogenesis, tumor cell intravasation, and suppress the anti-tumor

348 immune response(48). Our results expand this perspective to include a key role for early

349 infiltrating macrophages in initiating a collagen crosslinking and stiffening program that

350 ultimately fosters tumor aggression and progression. We also determined that TN breast

351 cancers contain much higher amounts of LH2- and LOX-derived collagen crosslinks.

352 Specifically, we demonstrated that significantly higher levels of HLCC crosslinks could explain

353 their higher stromal stiffness and aggressiveness(2). Intriguingly, in depth collagen analysis

354 suggested that each breast tumor subtype exhibits a distinct collagen organization, stiffness and

355 crosslinking profile, raising the possibility that distinct collagen architectures and crosslinking

356 signatures may reflect differences in tissue pathobiology. Indeed, TN breast cancers often

357 present with high macrophage infiltration; an observation that is consistent with our AFM, two

358 photon and collagen crosslinking analysis data(2). Given that the immune cell infiltrate

359 significantly influences treatment response it is tempting to speculate that distinct collagen

360 architectures, crosslinking signatures and stiffnesses similarly regulate therapeutic efficacy. 
We determined that stromal $\mathrm{LH} 2$ is a robust predictor of survival in breast cancer patients, especially in those that are lymph node positive, supporting a potentially important clinical link to stromal collagen crosslinking. Our data also revealed that both the upregulation of crosslinking enzymes and in turn, collagen crosslinking, occur at an early stage of malignancy that is concurrent with tumor cell invasion and coincident with the accumulation of infiltrating macrophages(49). In part, this may explain why therapeutics targeting LOX and LOXL2 have

367 thus far failed to significantly prolong cancer patient survival. Substantial levels of collagen 368 crosslinking are likely to have occurred prior to therapy administration, and while these agents 369 may prevent further crosslinking activity, they are not capable of reversing crosslinks or collagen 370 modifications already present in the tissue. The early initiation of collagen crosslinking also 371 suggests that a detectable increase in stromal LH2 may provide an early prognostic marker of 372 disease progression and aggression that could inform treatment strategies. To that end, our 373 results show for the first time that LH2-derived collagen crosslinks are not only a distinct feature

374 of enhanced stromal stiffening in TN breast cancer but are able to predict distant metastasis-

375 free survival. These results also suggest that the profile of collagen crosslinks, and not simply 376 crosslink abundance alone, may play a role in promoting tumor aggression, thus warranting 377 further investigations into the contributions of HLCCs to ECM mechanical properties and tumor 378 aggression.

379 Our findings identify fibroblasts as the dominant cell population that promotes

380 reorganization and crosslinking of interstitial collagen to stiffen the tissue stroma. Only stromal-

381 targeted, and not epithelial-targeted, tissue-specific inducible LOX produced any measurable 382 change in collagen architecture, stiffness or crosslink abundance in a spontaneous tumor $383(25,50,51)$. Our conclusion is consistent with earlier work in which we failed to detect any 384 alterations in fibrosis, collagen organization or stiffness when LOXL2 was either genetically385 ablated, or ectopically-increased in the mammary epithelium of PyMT mouse tumors, despite 386 documenting a significant impact on metastasis(40). Nevertheless, it is possible that our bulk 
assay would not detect any small increase in collagen crosslinking induced by invading tumor cells expressing high levels of LOX. To this end, immortalized tumor cells engineered to overexpress LOXL2 injected orthotopically as a bolus of cells were able to induce fibrosis and quantifiable changes in collagen remodeling and tissue stiffening; a finding we too confirmed using isolated PyMT tumor cells overexpressing the LOXL2 enzyme(40). Nevertheless, our data support the notion that in spontaneous tumors, the proximity of stromal cells to collagen and

393 their significantly higher expression of crosslinking enzymes dictate the profile and extent of 394 collagen crosslinking. Given that LH2 modifies collagen intracellularly and stromal cells secrete

395 the vast majority of the interstitial fibrillar collagen, it is not surprising that this is the case $(52,53)$.

396 Moreover, our results stress the necessity of choosing an appropriate model to study tumor associated ECM remodeling and suggest that orthotopic models may fail to accurately recapitulate the natural evolution of ECM in tumor progression.

401 the molecular nature of collagen modifications that accompany pathological fibrosis. Clarifying

402 the nature of collagen modifications and specific mechanisms regulating those alterations in

403 fibrosis will assist in the strategic design of novel, efficacious strategies to combat progressive

404 fibrosis and should prove instrumental in enabling further experimentation to understand

405 pathological fibrosis. Indeed, defining the molecular regulators that stimulate collagen cross-

406 linking, and mechanisms that distinguish resolvable from non-resolvable fibrosis would identify

407 attractive therapeutic targets for several pathological fibrotic diseases with limited treatment

408 options.

\section{Methods:}

\section{Human breast specimen acquisition and processing}

411 Fresh human breast specimens from breast reduction, prophylaxis, or breast tumor mastectomy

412 were either embedded in an optimum cutting temperature (OCT) aqueous embedding 
413 compound (Tissue-Plus, Scigen, Cat\# 4583) within a disposable plastic base mold (Fisher, Cat\#

414 22-363-554) and were snap frozen by direct immersion into liquid nitrogen and kept at $-80^{\circ} \mathrm{C}$

415 freezer until cryo-sectioning for analysis, or specimens were formalin fixed and paraffin

416 embedded (FFPE). All human breast specimens were collected from prospective patients

417 undergoing surgical resection at UCSF or Duke University Medical Center between 2010 and

418 2014. The selected specimens were de-identified, stored, and analyzed according to the

419 procedures described in Institutional Review Board (IRB) Protocol \#10-03832 and \#10-05046,

420 approved by the UCSF Committee of Human Resources and the Duke's IRB (Pro00034242)(2).

\section{$421 \quad$ Mouse studies}

422 Macrophages were depleted in MMTV-PyMT mice by i.p. injections of $1 \mathrm{mg}$ of anti-CSF1

423 antibody clone $5 \mathrm{~A} 1$ or an IgG1 control every 7 days starting at 4 weeks of age. Mice were

424 sacrificed at 8 and 11 weeks of age for tissue analysis.

425 Minoxidil and PBS vehicle was administered by i.p. injections of $3 \mathrm{mg} / \mathrm{kg}$ minoxidil in PBS three

426 times per week starting at 6 weeks of age. Mice were sacrificed at 11-12 and 13-14 weeks of

427 age for tissue analysis.

\section{Generation of mice}

429 All mouse studies were maintained under pathogen-free conditions and performed in

430 accordance with the Institutional Animal Care and Use Committee and the Laboratory Animal

431 Research Center at the University of California, San Francisco.

432 TetO-mLOX-eGFP construct and transgenic mouse generation

433 Full length mouse Lox (mLox) cDNA was purchased from OriGene. The full length ORF was

434 amplified by PCR using forward and reverse primers respectively:

435 GCAGGGATCCGCCACCATGCGTTTCGCCTGGGCTG and

436 GGCGTCTAGAGCACCATGCGTTTCGCCTGGGCTGTGC. Following digestion with BamHI

437 and Xbal, the PCR product was inserted into pSK TetO IRES 3xnlsEGFP(54) downstream of

438 the Tet regulated minimal CMV promoter and a 5' UTR containing a chimeric intron of human $\beta$ - 
globin and immunoglobulin heavy chain genes, which was expressed as a bicistronic mRNA via an internal ribosome entry site (IRES2) with eGFP targeted to the nucleus by a $\mathrm{N}$ terminal in

441 frame fusion of 3 tandem repeats of the SV40 nuclear localization sequence (nls). The fragment

442 containing the expression cassette from Tet regulated promoter to SV40 polyadenylation signal

443 (SV40pA) was agarose gel purified from Xhol- Eagl digested donor plasmid and was used to

444 generate TetO-mLox-eGFP transgenic mice by pronuclear injection into FVB/n oocyte (Mouse

445 Biology Program at UC Davis; project number MBP-834; colony number PN663).

\section{Generation of MMTV-PyMT/Col1a1(2.3)-tTA/TetO-mLox-eGFP mice}

447 The MMTV-PyMT/Col1a1(2.3)-tTA/TetO-Rs1 triple transgenic mice were generated by

448 heterozygote or homozygote crosses of mice carrying the TetO-mLox-eGFP transgene with

449 mice carrying heterozygote of the Col1a1(2.3)-tTA transgene (line 139)(55) or MMTV-rtTA and

450 MMTV-PyMT transgene(56) to generate the experimental triple-transgenic genotype. In all

451 breeding thereafter, MMTV-PyMT/Col11a1-tTA or MMTV-rtTA/TetO-mLox-eGFP male mice

452 were crossed with TetO-mLox-eGFP female mice. Two mg/mL doxycycline hyclate (Alfa Aesar;

453 Cat\# J60579) was added to 5\% sucrose water to modulate TetO-mLox-eGFP transgene

454 expression.

455 Preparation of human breast specimens for hydrolysis

456 OCT was removed from tissue blocks by first transferring biospecimens to a conical tube and

457 then performing $5 \mathrm{X}$ washes with $70 \%$ ethanol followed by $5 \mathrm{X}$ washes with $18 \mathrm{M} \Omega ~_{2} \mathrm{O}^{48}$. Each

458 wash consisted of vortexing the sample for 15 minutes at $4^{\circ} \mathrm{C}$ and then centrifuging at $18,000 \mathrm{x}$

$459 \mathrm{~g}$ for 15 minutes at $4^{\circ} \mathrm{C}$. Between 1 and 3 milligrams of tissue was washed with $1 \mathrm{X}$ PBS buffer

460 by vortexing for 15 minutes at $4^{\circ} \mathrm{C}$ and then sonicated on ice for 20 seconds using a Sonic

461 Dismembrator M100 (ThermoFisher, San Jose, CA, USA). The homogenate was then

462 centrifuged at $18,000 \times \mathrm{g}$ for 20 minutes at $4^{\circ} \mathrm{C}$. The supernatant was removed and the pellet

463 was re-suspended in $1 \mathrm{mg} / \mathrm{mL} \mathrm{NaBH}_{4}$ (prepared in $0.1 \mathrm{~N} \mathrm{NaOH}$ ) in $1 \mathrm{X}$ PBS for 1 hour at $4^{\circ} \mathrm{C}$ with

464 vortexing. The reaction was neutralized by adding glacial acetic acid to a final concentration of 

supernatant was removed and the pellet was washed three times with $18 \mathrm{M} \Omega \mathrm{H}_{2} \mathrm{O}$ to remove residual salt that could interfere with downstream LC-MS/MS analysis. The remaining pellet was dried under vacuum for further analysis.

\section{Protein hydrolysis}

470 The dried sample was placed in a glass hydrolysis vessel and hydrolyzed in $6 \mathrm{~N} \mathrm{HCl}, 0.1 \%$

471 phenol. The hydrolysis vessel is flushed with N2 gas, sealed and placed in a $110^{\circ} \mathrm{C}$ oven for 24

472 hours. After hydrolysis, the sample was cooled to room temperature and then placed at $-80^{\circ} \mathrm{C}$

473 for 30 minutes prior to lyophilization. The dried sample was re-hydrated in $100 \mu \mathrm{L}$ of $18 \mathrm{M} \Omega \mathrm{H}_{2} \mathrm{O}$

474 for 5 minutes, then $100 \mu \mathrm{L}$ of glacial acetic acid for 5 minutes and finally $400 \mu \mathrm{L}$ of butan-1-ol for

4755 minutes. Importantly, $10 \mu \mathrm{L}$ of sample is removed after re-hydration in water and saved for

476 determination of hydroxyproline content.

\section{Preparation of crosslink enrichment column}

478 CF-11 cellulose powder is loaded in a slurry of butan-1-ol: glacial acetic acid, water (4:1:1)

479 solution onto a Nanosep MF GHP $0.45 \mu \mathrm{m}$ spin columns until a settled resin bed volume of

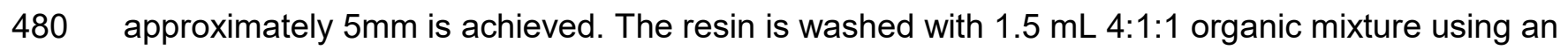

481 in-house vacuum manifold set up. Re-hydrated samples are then loaded onto individual

482 columns, the vacuum is turned on and the sample is pulled through the resin into glass

483 collection vials. The flow through is again passed over the resin to ensure maximal binding of

484 crosslinked amino acids and set aside. The column is then washed with $1.5 \mathrm{~mL}$ of fresh $4: 1: 1$

485 organic mixture. A fresh collection vessel is placed under the column and $750 \mu \mathrm{L}$ of $18 \mathrm{M} \Omega \mathrm{H} 2 \mathrm{O}$

486 is used to elute crosslinked amino acids off of the CF-11 resin. The eluent is then placed in a

487 speed vac and run until complete dryness. Dried eluent is then reconstituted in a buffer

488 appropriate for downstream MS analysis on amide HILIC UHPLC columns.

\section{UHPLC analysis}


490 Up to $20 \mu \mathrm{L}$ of tissue hydrolysates were analyzed on a Vanquish UPHLC system

491 (ThermoFisher, San Jose, CA, USA) using an Acquity UHPLC BEH Amide column (2.1 x

$492100 \mathrm{~mm}, 1.7 \mu \mathrm{m}$ particle size - Waters, Milford, MA, USA). Samples were separated using a 5

493 minute gradient elution (55\% - 40\% Mobile phase B) at $250 \mu \mathrm{L} / \mathrm{min}$ (mobile phase: (A) $10 \mathrm{mM}$

494 ammonium acetate adjusted to $\mathrm{pH} 10.2$ with $\mathrm{NH}_{4} \mathrm{OH}$ (B) 95\% acetonitrile, 5\% Mobile Phase A,

$495 \mathrm{pH} 10.2$, column temperature: $35^{\circ} \mathrm{C}$.

\section{MS data acquisition}

497 The Vanquish UPHLC system (ThermoFisher, San Jose, CA, USA) was coupled online with a

498 QExactive mass spectrometer (Thermo, San Jose, CA, USA), and operated in two different

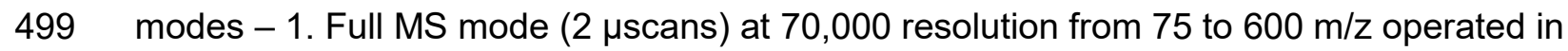

500 positive ion mode and 2. PRM mode at 17,500 resolution with an inclusion list of in-tact

501 crosslinked amino acid masses (Supplementary Table 2), and an isolation window of $4 \mathrm{~m} / \mathrm{z}$.

502 Both modes were operated with $4 \mathrm{kV}$ spray voltage, 15 sheath gas and 5 auxiliary gas.

503 Calibration was performed before each analysis using a positive calibration mix (Piercenet -

504 Thermo Fisher, Rockford, IL, USA). Limits of detection (LOD) were characterized by

505 determining the smallest injected crosslinked amino acids (LNL, DHLNL, d-Pyr, ) amount

506 required to provide a signal to noise $(\mathrm{S} / \mathrm{N})$ ratio greater than three using $<5 \mathrm{ppm}$ error on the

507 accurate intact mass. Based on a conservative definition for Limit of Quantification (LOQ), these

508 values were calculated to be threefold higher than determined LODs.

\section{MS data analysis}

510 MS Data acquired from the QExactive were converted from a raw file format to $\mathrm{mzXML}$ format

511 using MassMatrix (Cleveland, OH, USA). Assignment of crosslinked amino acids was performed

512 using MAVEN (Princeton, NJ, USA)(58). The MAVEN software platform provides the means to

513 evaluate data acquired in Full MS and PRM modes and the import of in-house curated peak lists

514 for rapid validation of features. Normalization of crosslinked amino acid peak areas was

515 performed using two parameters, 1. Hydroxy proline content and 2. Tissue dry weight pre- 
516 hydrolysis (in milligrams)(57). Hydroxy proline content is determined by running a 1:10 dilution

517 of the pre-enrichment sample through the Full MS mode (only) described above and exporting

518 peak areas for each run.

\section{Quantification of crosslinked amino acids}

520 Relative quantification of crosslinked amino acids was performed by exporting peak areas from

521 MAVEN into GraphPad (La Jolla, CA, USA) and normalizing based on the two parameters

522 described above. Statistical analysis, including T test and ANOVA (significance threshold for P

523 values $<0.05$ ) were performed on normalized peak areas. Total crosslink plots were generated

524 by summing normalized peak areas for all crosslinks in a given sample. Total HLCC plots were

525 generated by summing normalized peak areas for all HLCC (DHLNL, Pyr, dPyr) crosslinks in a

526 given sample.

\section{Picrosirius red staining and quantification}

528 FFPE tissue sections were stained using 0.1\% Picrosirius red (Direct Red 80, Sigma-Aldrich,

529 Cat\# 365548 and picric acid solution, Sigma-Aldrich, Cat\# P6744) and counterstained with

530 Weigert's hematoxylin (Cancer Diagnostics, Cat\# CM3951), as previously described(2).

531 Polarized light images were acquired using an Olympus IX81 microscope fitted with an analyzer

532 (U-ANT) and a polarizer (U-POT, Olympus) oriented parallel and orthogonal to each other.

533 Images were quantified using an ImageJ macro to determine percentage area coverage per

534 field of view. The ImageJ macro is available at https://github.com/northcottj/picrosirius-red.

\section{Second harmonic generation image acquisition}

536 Second harmonic generation (SHG) imaging was performed using a custom-built two-photon

537 microscope setup equipped resonant-scanning instruments based on published designs

538 containing a five-PMT array (Hamamatsu, C7950), as previously published(2). The setup was

539 used with two channel simultaneous video rate acquisition via two PMT detectors and an

540 excitation laser (2W MaiTai Ti-Sapphire laser, 710-920 nm excitation range). SHG imaging was

541 performed on a Prairie Technology Ultima System attached to an Olympus BX-51 fixed stage 
542 microscope equipped with a 25X (NA 1.05) water immersion objective. Paraformaldehyde-fixed

543 or FFPE tissue sections were exposed to polarized laser light at a wavelength of $830 \mathrm{~nm}$ and

544 emitted light was separated using a filter set (short pass filter, $720 \mathrm{~nm}$; dichroic mirror, $495 \mathrm{~nm}$;

545 band pass filter, $475 / 40 \mathrm{~nm}$ ). Images of $x-y$ planes at a resolution of $0.656 \mathrm{~mm}$ per pixel were

546 captured using at open-source Micro-Magellan software suite.

\section{Immunofluorescence/Immunohistochemistry}

548 Immunofluorescence staining was performed as previously described(2). Briefly, mouse tissues

549 were harvested and fixed with 10\% buffered formalin phosphate (Fisher, Cat\# 100-20) for 16-24

550 hours at room temperature and then further processed and paraffin-embedded. Five- $\mu \mathrm{m}$

551 sections dried for 30 minutes in $60^{\circ} \mathrm{C}$, follow by deparaffinization and rehydration. Antigen

552 retrieval was perform using DAKO Target Retrieval Solution (DAKO, Cat\# S1699) for five

553 minutes in a pressure cooker set to high pressure. Tissue sections were incubated with anti-

554 FAK pY397 antibody (Abcam, Cat\# Ab39967, dilution 1:25) overnight at $4^{\circ} \mathrm{C}$ and with anti-rabbit

555 IgG Alex Fluor 633 (ThermoFisher, Cat\# A-21070, dilution 1:2000) for one hour at room

556 temperature. Antigen retrieval for immunofluorescent staining of SMAD2 pS465/467,

557 cytokeratin 8+18, cytokeratin 5, F4/80, vimentin, and PDGFRa was performed using Diva

558 Decloaker (BioCare, Cat\# DV2004MX) for five minutes in a pressure cooker set to high

559 pressure. Tissue sections were incubated with anti-SMAD2 pS465/467 antibody (Millipore, Cat\#

560 AB3849-I, dilution 1:100), anti-F4/80 antibody (AbD Serotec, clone Cl:A3-1, Cat\# MCA497GA,

561 dilution 1:400), anti-cytokeratin 8+18 antibody (Fitzgerald, Cat\# 20R-CP004, dilution 1:400),

562 anti-cytokeratin 5 antibody (Fitzgerald, Cat\# 20R-CP003, dilution 1:400), anti-vimentin antibody

563 (Cell Signaling, Cat\# 5741, dilution 1:100), and anti-PDGFRa (CD140a) antibody (Biolegend,

564 Cat\# 135901, dilution 1:100) overnight at $4^{\circ} \mathrm{C}$ and with anti-rat IgG Alex Fluor 488

565 (ThermoFisher, Cat\# A-11006, dilution 1:1000), anti-guinea pig IgG Alex Fluor 568

566 (ThermoFisher, Cat\# A-11075, dilution 1:1000), anti-rabbit IgG Alex Fluor 633 (ThermoFisher, 
567 Cat\# A-21070, dilution 1:2000), anti-rabbit IgG Alex Fluor 633 (ThermoFisher, Cat\# A-21070,

568 dilution 1:1000) for one hour at room temperature.

569 Quantification of stromal nuclear SMAD2 pS465/467 was performed using Imaris 9. Surfaces

570 were created around each nucleus and epithelial nuclei were manually excluded based on

571 cytokeratin signal and cell morphology. The means of the mean nuclear signal intensity for all

572 stromal nuclei were calculated for each field of view and averaged for every animal.

573 Lungs from 11 week old IgG1 control and anti-CSF1 treated PyMT mice were cut into 5 micron

574 sections from 5 layers with 100 microns between the first three layers and 50 microns between

575 the last two layers. Sections were analyzed for metastases by PyMT staining. Antigen retrieval

576 was performed in Tris-EDTA buffer at $\mathrm{pH} 9$ for four minutes in a pressure cooker set to low

577 pressure. Tissue sections were incubated with anti-PyMT antibody (Novus Biologicals, Cat\# NB-

$578100-2749$, dilution 1:250) overnight at $4^{\circ} \mathrm{C}$ and with a biotinylated anti-rat antibody for 1 hour at

579 room temperature. Vectastain Elite ABC (Vector, Cat\# PK6100) and ImmPACT DAB Peroxidase

580 (Vector, Cat\# SK-4105) were used for signal detection and nuclei were counterstained with

581 methyl green.

\section{2 mRNA In Situ Hybridization}

583 Fresh, RNase-free FFPE sections were stained with RNAscope multiplex fluorescent reagent kit

584 V2 according to standard manufacturer protocol. Target retrieval was performed using 8 minute

585 incubation in a pressure cooker set to low pressure. Opal 520 and Opal 570 (PerkinElmer) were

586 used at 1:1500 for target visualization.

\section{Gene expression by RT-qPCR}

Total RNA was reverse-transcribed using random primers (Amersham Bioscienes) and results were normalized to $18 \mathrm{~S}$ RNA to control for varying cDNA concentration between samples. The primer sequences used are 18s forward 5'-GGATGCGTGCATTTATCAGA-3' and reverse 5'GGCGACTACCATCGAAAGTT-3', Lox forward 5'-CGGGAGACCGTACTGGAAGT-3' and 
reverse 5'-CCCAGCCACATAGATCGCAT-3', Loxl2 forward 5'-CACAGGCACTACCACAGCAT3' and reverse 5'-CCAAAGTTGGCACACTCGTA-3', and Tgfb1 forward 5'TCATGTCATGGATGGTGCCC-3' and reverse 5'-GTCACTGGAGTTGTACGGCA-3'.

\section{Atomic force microscopy data acquisition}

589 Atomic force microscopy (AFM) measurements were performed as previously described(2).

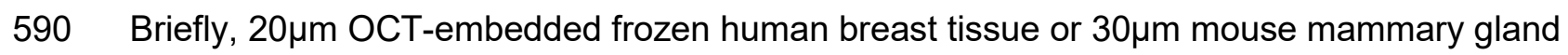
591 sections were fast thawed by immersion in PBS at room temperature. Next, these sections were 592 immersed in PBS containing phosphatase inhibitors (Roche, Cat\# 04906845001), protease 593 inhibitor (Roche, Cat\# 04693124001), and propidium iodide (ACROS, Cat\# 440300250) and 594 placed on the stage for AFM measurements. AFM indentations were performed using an 595 MFP3D-BIO inverted optical AFM (Asylum Research) mounted on a Nikon TE2000-U inverted

596 fluorescent microscope. Silicon nitride cantilevers were used with a spring constant of $0.06 \mathrm{~N} \mathrm{~m}^{-}$

$597{ }^{1}$ and a borosilicate glass spherical tip with $5 \mu \mathrm{m}$ diameter (Novascan Tech). The cantilever was 598 calibrated using the thermal oscillation method prior to each experiment. The indentation rate 599 was held constant within each study but varied between 2-20 $\mu \mathrm{ms}^{-1}$ with a maximum force of 2 $600 \mathrm{nN}$ between studies. Force maps were obtained as a raster series of indentations utilizing the 601 FMAP function of the IGOR PRO build supplied by Asylum Research. Elastic properties of ECM 602 were reckoned using the Hertz model. A Poisson's ratio of 0.5 was used in the calculation of the 603 Young's elastic modulus.

\section{Western blotting}

605 Snap frozen tissues were ground while frozen and lysed in $2 \%$ SDS containing protease and 606 phosphatase inhibitor. Samples were boiled for 5 minutes $\left(95^{\circ} \mathrm{C}\right)$ and loaded onto the SDS607 polyacrylamide gel, and protein was separated at 110 constant volts.

608 The protein was transferred onto a pre-wet polyvinylidene difluoride (PVDF) membrane (100\% 609 methanol, 1 minute) at $250 \mathrm{~mA}$ for 2 hours. The PVDF membrane was rinsed with TBST and 
610 non-specific binding was blocked with $5 \%$ nonfat dry milk dissolved in TBST. The membrane

611 was then incubated with the primary antibody overnight at $4^{\circ} \mathrm{C}$, washed with TBST, incubated

612 with horseradish-peroxidase conjugated secondary antibody (1 hour, room temperature; dilution

613 1:5000), washed with TBST, and detected with the chemiluminescence system Quantum HRP

614 substrate (Advansta \#K-12042). Quantification was performed using gel densitometry in

615 ImageJ. Primary antibodies used are anti-LOX (1:1000, Abcam Cat\# ab174316) and anti-E-

616 cadherin (1:1000, Cell Signlaing Cat\#3195).

\section{Flow cytometry}

618 Mouse tissue was harvested and chopped with a razor blade. Chopped tissue was digested in

$619100 \mathrm{U} / \mathrm{mL}$ Collagenase Type 1 (Worthington Biochemical Corporation, Cat\# LS004196), 500

$620 \mathrm{U} / \mathrm{mL}$ Collagenase Type 4 (Worthington Biochemical Corporation, Cat\# LS004188), and 200

$621 \mu \mathrm{g} / \mathrm{mL}$ DNase I (Roche, Cat\# 10104159001) while shaking at $37^{\circ} \mathrm{C}$. Digested tissue was filtered

622 using a $100 \mu \mathrm{m}$ filter to remove remaining pieces. Red blood cells were lysed in ammonium-

623 chloride-potassium buffer and remaining cells were counted. Cells were stained with

624 fluorophore-conjugated primary antibodies for 30 minutes on ice and subsequently stained with

625 a viability marker. Antibodies used for staining were anti-mouse CD24-PE (BD Pharmingen,

626 Cat\# 553262), anti-mouse TER-119-APC (BioLegend, Cat\# 116212), anti-mouse CD45-APC

627 (BioLegend, Cat\# 103112), anti-mouse CD29-AF700 (BioLegend, Cat\# 102218), anti-mouse

628 Ly6G-BV421 (BioLegend, Cat\# 127628), anti-mouse F4/80-BV510 (BioLegend, Cat\# 123135),

629 anti-mouse CD29-AF488 (BioLegend, Cat\# 102212), anti-mouse CD140a-PE (BioLegend, Cat\#

630 135906), anti-mouse CD31-APC (BioLegend, Cat\# 102410), anti-mouse CD11C-BV605 (BD

631 Pharmingen, Cat\# 563057), anti-mouse CD24-BV650 (BD Pharmingen, Cat\# 563545), anti-

632 mouse CD11b-PerCP-Cy5.5 (eBiosciences, Cat\# 45-0112-82), anti-mouse CD45-AF700

633 (BioLegend, Cat\# 103128), anti-mouse Ly6C-BV711 (BioLegend, Cat\# 128037), anti-mouse 
634 MHCII-PE-Cy7 (BioLegend, Cat\# 107630), and Zombie NIR Fixable Viability Dye (BioLegend,

635 Cat\# 423105). Cells were then analyzed on a flow cytometer.

636 Patient gene expression analysis

637 For the stroma and epithelium specific gene expression analysis, the breast cancer datasets

638 from Finak et al. 2008 and Gruosso et al. 2019 have been used. Briefly, whole Human Genome

$63944 \mathrm{~K}$ arrays (Agilent Technologies, product G4112A) were used for stroma and epithelial

640 expression profiles. Details of laser capture microdissection, RNA extraction, labeling,

641 hybridization, scanning and quality filters are described in Finak et al., 2006 and 2008. Briefly,

642 the dataset was normalized using loess (within-array) and quantile (between-array)

643 normalization. Probes were ranked by Inter-quartile range (IQR) values, and the most variable

644 probe per gene across expression data were selected for further analysis. Replicate arrays with

645 a concordance above 0.944 were averaged before assessing differential expression.

646 An association between PLOD2 and distant metastasis-free survival (DMFS) has been

647 determined using an online tool (http://xena.ucsc.edu) to download GEO data (GSE2034,

648 GSE5327, and GSE7390) from 683 patients analyzed on Affymetrix U133A platform as

649 described in Yau et al.(46). Patients have been excluded from analyses if their molecular

650 subtyping of ER/HER2 status and PAM50 did not align: ER+/HER2- must always be luminal,

651 ER+ or-/HER2+ must be HER2+, and ER-/HER2- must always be basal-like. PLOD2 expression

652 levels have been divided based on the median for each tumor subtype: ER+/HER2- (low n=157;

653 high=157), ER- or +/HER2+ (low n=36; high=37), and ER-/HER2- (low n=66; high=67). All

654 statistical analyses were done using GraphPad Prism Version 6.01: Kruskal-Wallis one-way

655 ANOVA test was applied to assess the relationship in PLOD2 expression levels among tumor

656 subtypes and log rank P value (Mantel-Cox) tests for DMFS curves.

657 An association between PLOD2 gene expression and relapse-free survival (RFS) has been

658 determined using an online tool (http://kmplot.com/analysis/) from 1,809 patients analyzed on 

202620 were used for PLOD2 probes (2014 version) in these analyses. All breast cancer

661 patients in this database were included regardless to lymph node status, TP53 status, or grade.

662 No restrictions were placed in term of patient treatment. PLOD2 expression levels have been

663 divided based on the median for each tumor subtype: ER+/PR+/HER2- (low n=170; high=169),

664 ER-/PR-/HER2+ (low n=58; high=57), and ER-/PR-/HER- (low n=128; high=127). Hazard ratio

665 (and 95\% confidence intervals) and log rank $P$ values were calculated and displayed once the 666 data were plotted using the online tool.

667 The cBioPortal for Cancer Genomics was used to determine the levels of LOX, PLOD2, and

668 LOXL2 gene expression in breast cancer patients segregated by ER and HER2 status(59,60).

669 ER, PR, and HER2 status were determined by gene expression levels. Samples positive for

670 both ER/PR and HER2 overexpression were excluded from subtype analysis. The cBioPortal

671 was also used to assess gene expression associations of LOX, PLOD2, and LOXL2 with HIF1A,

672 CCL2, CD163, and CD68. All data accessed via cBioPortal are from the 1904 patients in the

673 METABRIC dataset analyzed for gene expression by Illumina human v3 microarray(61). All

6741904 samples were included in correlation analyses.

\section{Statistical analysis}

676 GraphPad Prism Version 6.01 was used to perform all statistical analyses with the exception of

677 LH2 IHC and RFS correlations with PLOD2. Statistical significance was determined using the

678 appropriate tests as noted in the figure legends or method section.

\section{LH2 IHC and prognostic analyses:}

680 Study population

681 The female Malmö Diet and Cancer Study (MDCS) cohort consists of women born 1923-

682 1950)(62,63). Information on incident breast cancer is annually retrieved from the Swedish

683 Cancer Registry and the South Swedish Regional Tumor Registry. Follow-up until December

68431,2010 , identified a total of 910 women with incident breast cancer, the following conditions 
685 excluded patients: 1 ) with in situ only cancers $(n=68), 2)$ who received neo-adjuvant treatments

$686(n=4), 3)$ with distant metastasis at diagnosis $(n=14), 4)$ those who died from breast cancer-

687 related causes $\leq 0.3$ years from diagnosis $(n=2)$, and finally 5$)$ patients with bilateral cancers

688 ( $n=17)$. In addition, one patient who declined treatment for four years before accepting surgery

689 was excluded. Patient characteristics at diagnosis and pathological tumor data were obtained

690 from medical records. Information on cause of death and vital status was retrieved from the

691 Swedish Causes of Death Registry, with last follow-up December 31st, 2014. Ethical permission

692 was obtained from the Ethical Committee at Lund University (Dnr 472/2007). All participants

693 originally signed a written informed consent form.

694 Tumor evaluation

695 Tumor samples from incident breast cancer cases in MDCS were collected, and a tissue

696 microarray (TMA) including two 1-mm cores from each tumor was constructed (Beecher, WI,

697 USA). Within the study population $(\mathrm{N}=910)$, tumor tissue cores were accessible from 718

698 patients. Four- $\mu \mathrm{m}$ sections dried for one hour in $60^{\circ} \mathrm{C}$ were automatically pretreated using the

699 Autostainer plus, DAKO staining equipment with Dako kit K8010 (Dako, DK). A primary mouse

700 monoclonal Lysyl Hydroxylase 2 (LH2) antibody (Origene; Cat\# TA803224, dilution 1:150) was

701 used for the immunohistochemical staining.

702 TMA cores were analyzed by a cohort of 4 anatomic pathologists (ACN, AC, JG, AN) using the

703 PathXL digital pathology system (http://www.pathxl.com, PathXL Ltd., UK) blinded to all other

704 clinical and pathologic variables. Immunohistochemistry for LH2 was assessed separately for

705 stromal and neoplastic epithelial components of the tumors. Stromal LH2 staining was assessed

706 with the semi-quantitative $\mathrm{H}$-score which combines intensity and proportion positive

707 assessments into a continuous variable from 0-300(64). Cellular stromal components were

708 assessed (including fibroblasts, macrophages, endothelial cells, adipocytes, and other stromal

709 cell types) while areas of significant lymphocytic infiltrate were specifically excluded from the

710 percent positive estimation. Neoplastic epithelial LH2 staining intensity was scored 0-3+ based 
711 on the predominant intensity pattern in the tumor — invasive tumor cells did not display

712 significant intra-tumoral heterogeneity of LH2 staining within each core. Verification of inter-

713 observer reproducibility for the $\mathrm{H}$-score was established in a training series of 16 cases

714 evaluated by all study pathologists to harmonize scoring. Inter-observer agreement in the

715 training set was very high, evaluating the IHC scores both as continuous variables (Pearson

716 correlation coefficients ranging from $0.912-0.9566$, all $p$ values $<0.0001$ ), and after

717 transformation into categorical data (negative, low, and moderate/high; weighted kappa

718 coefficients ranging from $0.673-0.786)$. In addition, 50 cases of the study cohort were evaluated

719 blindly by two pathologists to confirm data fidelity; the Pearson correlation coefficient $=0.7507$

$720 \quad(p=5.7$ E-05), considered a strong level of agreement.

721 After exclusion of cases for which LH2 was not evaluable on the TMA, H-scores for 505 patients

722 were included for statistical associations with clinicopathologic features and patient outcome.

723 Each patient was represented by two cores, and TMA core 1 and core 2 were merged into a

724 joint variable favoring the highest stromal LH2 H-score or epithelial LH2 intensity because we

725 predict that higher $\mathrm{H}$-score would drive patient outcome in accordance with our gene expression

726 data demonstrating high LH2 expression correlated with poor outcome. The Pearson correlation

727 coefficient between cores $=0.647$, demonstrating moderate agreement among the stromal LH2

$728 \mathrm{H}$-scores for the two cores. In cases with only one TMA core providing a LH2 score, the

729 expression of this core was used. Further, the joint stromal LH2 variable was categorized into

730 tertiles based on the study population with valid $\mathrm{LH} 2$ annotation $(\mathrm{N}=505)$. The lowest tertile of

$731 \mathrm{LH} 2 \mathrm{H}$-scores were defined as scores between 0 and less or equal to $120(\mathrm{~N}=171)$, the

732 intermediate $\mathrm{H}$-score as above 120 and equal or less than $230(\mathrm{~N}=188)$, and the highest stromal

733 LH2 score as above $230(\mathrm{~N}=146)$.

734 Statistical analyses for LH2 IHC analyses

735 Patient and tumor characteristics at diagnosis in relation to stromal LH2 expression were

736 categorized and presented as percentages. Continuous variables are presented as the mean 
737 and $\mathrm{min} / \mathrm{max}$. The associations between LH2 expression and grade or tumor size, respectively,

738 were analyzed through linear-by-linear association. The association between LH2 expression

739 and prognosis was examined using breast cancer-specific mortality as endpoint, which was

740 defined as the incidence of breast cancer-related death. Follow-up was calculated from the date

741 of breast cancer diagnosis to the date of breast cancer-related death, date of death from

742 another cause, date of emigration or the end of follow-up as of December 31st, 2014. Main

743 analyses included the overall population; additional analyses were performed in subgroup

744 analyses stratified by estrogen receptor (ER) or axillary lymph node involvement (ALNI) status.

745 The prognostic impact of stromal LH2 expression was analyzed through Cox proportional

746 hazards analyses, which yielded hazard ratios $(\mathrm{HR})$ and $95 \%$ confidence intervals $(\mathrm{Cl})$ for crude

747 models, and multivariate models adjusted for age at diagnosis (model 1) and tumor

748 characteristics ER (dichotomized, cut-off $10 \%$ stained nuclei), ALNI (none or any positive lymph

749 node involvement), histological grade (Nottingham grade I-III), and tumor size (dichotomized

750 using cut-off $20 \mathrm{~mm}$ ). Kaplan-Meier curves including the LogRank test indicated LH2 status to

751 particularly impact the first 10 years after diagnosis and survival variables constructed to

752 capture these effects were used in Cox regression models investigating the effects during the

753 first post-diagnostic decade. All statistical analyses were performed in SPSS version 22.0

754 (IBM). 


\section{Author Contributions}

757 V.M.W., K.C.H., O.M., A.S.B., and A.P.D. conceived the project, prepared figures and wrote the

758 manuscript. A.S.B, T.P., T.N., and K.C.H. developed the xAAA method and A.S.B performed all

759 LC-MS and LC-PRM experiments. J.N.L generated the TetO_mLOX mouse model. O.M.

760 designed and conducted in vivo experiments using inducible LOX overexpression models. B.R.

761 and L.C. designed and conducted CSF1 blocking antibody mouse experiment. O.M. and A.P.D.

762 performed and quantified immunofluorescence, H\&E, PS and SHG imaging and analyses on

763 mouse tissue samples. I.A., A.P.D., and J.M.B performed AFM on human or mouse tissue

764 specimens. I.A. performed SHG imaging on human tissue. E.S.H, and P.K. provided human

765 breast tumor biopsies for XAAA. B.R. and L.C. designed and conducted immunoprofiling on

766 human breast tumor via flow cytometry. O.M. and A.P.D. performed all gene expression

767 analyses with the exception of Fig. 5g-j. T.G, H.K. and M.P. performed gene expression

768 analyses gene expression in microdissected epithelial and stromal compartments of human

769 invasive breast carcinomas. O.M. and A.P.D. designed and conducted in vivo experiments

770 using minoxidil treatment. S.B. established and managed MDCS cohort used for LH2 IHC. Z.W.

771 and S.B. performed LH2 IHC. A.C.N. designed scoring schemes for stromal and neoplastic

772 epithelial LH2 IHC, and A.C.N, A.N., J.G., and A.C. scored all human biopsies. S.B., O.B.,

773 A.C.N., O.M., and V.M.W analyzed and interpreted clinical data from LH2 scores.

774 Acknowledgements:

775 We thank J. Northcott for writing the ImageJ Macro, L. Korets for mouse husbandry and N.

776 Korets for histology support, as well as K. Lövgren and S. Baker for LH2 immunostaining on

777 patient biopsies. The work was supported by investigator grants through the US National

778 Cancer Institute R33 CA183685 (K.C.H \& V.M.W) and R01CA192914 and CA174929 to

779 (V.M.W), and R01CA222508-01 to (V.M.W. and E.S.H.), as well as US DOD Breast Cancer

780 Research Program (BCRP) grant BC122990 (V.M.W). Trainee support was provided by US

781 DOD BCRP grant BC130501 (O.M.), US NIH grants TL1 TR001081 \& US NIH T32 HL007171 
US NIH R01 CA057621 (Z.W.) also supported the work.

\section{References:}

1. Laklai H, Miroshnikova YA, Pickup MW, Collisson EA, Kim GE, Barrett AS, et al. Genotype tunes pancreatic ductal adenocarcinoma tissue tension to induce matricellular fibrosis and tumor progression. Nat Med 2016;22(5):497-505 doi 10.1038/nm.4082.

2. Acerbi I, Cassereau L, Dean I, Shi Q, Au A, Park C, et al. Human breast cancer invasion and aggression correlates with ECM stiffening and immune cell infiltration. Integr Biol (Camb) 2015;7(10):1120-34 doi 10.1039/c5ib00040h.

3. Dvorak HF. Tumors: wounds that do not heal. Similarities between tumor stroma generation and wound healing. N Engl J Med 1986;315(26):1650-9 doi 10.1056/NEJM198612253152606.

4. Conklin MW, Eickhoff JC, Riching KM, Pehlke CA, Eliceiri KW, Provenzano PP, et al. Aligned collagen is a prognostic signature for survival in human breast carcinoma. The American journal of pathology 2011;178(3):1221-32 doi 10.1016/j.ajpath.2010.11.076.

5. Olive KP, Jacobetz MA, Davidson CJ, Gopinathan A, Mclntyre D, Honess D, et al. Inhibition of Hedgehog signaling enhances delivery of chemotherapy in a mouse model of pancreatic cancer. Science 2009;324(5933):1457-61 doi 10.1126/science.1171362.

6. Provenzano PP, Cuevas C, Chang AE, Goel VK, Von Hoff DD, Hingorani SR. Enzymatic targeting of the stroma ablates physical barriers to treatment of pancreatic ductal adenocarcinoma. Cancer Cell 2012;21(3):418-29 doi 10.1016/j.ccr.2012.01.007.

7. Netti PA, Berk DA, Swartz MA, Grodzinsky AJ, Jain RK. Role of extracellular matrix assembly in interstitial transport in solid tumors. Cancer Res 2000;60(9):2497-503.

8. Jain RK, Lee JJ, Hong D, Kurzrock R. Reply to A. Levy et al. J Clin Oncol 2013;31(3):396 doi 10.1200/JCO.2012.46.4867.

9. Egeblad M, Rasch MG, Weaver VM. Dynamic interplay between the collagen scaffold and tumor evolution. Curr Opin Cell Biol 2010;22(5):697-706 doi 10.1016/j.ceb.2010.08.015.

10. Chaudhuri O, Koshy ST, Branco da Cunha C, Shin JW, Verbeke CS, Allison KH, et al. Extracellular matrix stiffness and composition jointly regulate the induction of malignant phenotypes in mammary epithelium. Nat Mater 2014;13(10):970-8 doi 10.1038/nmat4009.

11. Provenzano PP, Inman DR, Eliceiri KW, Keely PJ. Matrix density-induced mechanoregulation of breast cell phenotype, signaling and gene expression through a FAK-ERK linkage. Oncogene 2009;28(49):4326-43.

12. Levental KR, Yu H, Kass L, Lakins JN, Egeblad M, Erler JT, et al. Matrix crosslinking forces tumor progression by enhancing integrin signaling. Cell 2009;139(5):891-906.

13. Mekhdjian AH, Kai F, Rubashkin MG, Prahl LS, Przybyla LM, McGregor AL, et al. Integrin-mediated traction force enhances paxillin molecular associations and adhesion dynamics that increase the invasiveness of tumor cells into a three-dimensional extracellular matrix. Mol Biol Cell 2017;28(11):1467-88 doi 10.1091/mbc.E16-09-0654.

14. Pickup MW, Laklai H, Acerbi I, Owens P, Gorska AE, Chytil A, et al. Stromally derived lysyl oxidase promotes metastasis of transforming growth factor-beta-deficient mouse mammary carcinomas. Cancer Res 2013;73(17):5336-46 doi 10.1158/0008-5472.CAN13-0012. 
827

828

829

830

831

832

833

834

835

836

837

838

839

840

841

842

843

844

845

846

847

848

849

850

851

852

853

854

855

856

857

858

859

860

861

862

863

864

865

866

867

868

869

870

871

872

873

874

875

15. Pickup MW, Mouw JK, Weaver VM. The extracellular matrix modulates the hallmarks of cancer. EMBO Rep 2014; 15(12):1243-53 doi 10.15252/embr.201439246.

16. Provenzano PP, Inman DR, Eliceiri KW, Knittel JG, Yan L, Rueden CT, et al. Collagen density promotes mammary tumor initiation and progression. BMC Med 2008;6:11 doi 1741-7015-6-11 [pii]

10.1186/1741-7015-6-11.

17. Eyre DR, Weis MA, Wu J-J. Advances in collagen cross-link analysis. Methods 2008;45(1):65-74.

18. Mammoto T, Ingber DE. Mechanical control of tissue and organ development. Development 2010;137(9):1407-20.

19. Yamauchi M, Shiiba M. Lysine hydroxylation and cross-linking of collagen. Posttranslational Modifications of Proteins 2008:95-108.

20. Yamauchi M, Sricholpech M. Lysine post-translational modifications of collagen. Essays in biochemistry 2012;52:113-33.

21. Miller BW, Morton JP, Pinese M, Saturno G, Jamieson NB, McGhee E, et al. Targeting the LOX/hypoxia axis reverses many of the features that make pancreatic cancer deadly: inhibition of LOX abrogates metastasis and enhances drug efficacy. EMBO Mol Med 2015;7(8):1063-76 doi 10.15252/emmm.201404827.

22. Erler JT, Weaver VM. Three-dimensional context regulation of metastasis. Clin Exp Metastasis 2009;26(1):35-49 doi 10.1007/s10585-008-9209-8.

23. Chen $\mathrm{Y}$, Terajima M, Yang Y, Sun L, Ahn Y-H, Pankova D, et al. Lysyl hydroxylase 2 induces a collagen cross-link switch in tumor stroma. The Journal of clinical investigation 2015; 125(3):1147.

24. Gilkes DM, Bajpai S, Wong CC, Chaturvedi P, Hubbi ME, Wirtz D, et al. Procollagen lysyl hydroxylase 2 is essential for hypoxia-induced breast cancer metastasis. Molecular cancer research 2013;11(5):456-66.

25. Erler JT, Bennewith KL, Nicolau M, Dornhofer N, Kong C, Le QT, et al. Lysyl oxidase is essential for hypoxia-induced metastasis. Nature 2006;440(7088):1222-6 doi 10.1038/nature04695.

26. Lampi MC, Reinhart-King CA. Targeting extracellular matrix stiffness to attenuate disease: From molecular mechanisms to clinical trials. Sci TransI Med 2018;10(422) doi 10.1126/scitransImed.aao0475.

27. Grivennikov SI, Greten FR, Karin M. Immunity, inflammation, and cancer. Cell 2010;140(6):883-99 doi 10.1016/j.cell.2010.01.025.

28. Mack M. Inflammation and fibrosis. Matrix Biol 2018;68-69:106-21 doi 10.1016/j.matbio.2017.11.010.

29. Wick G, Grundtman C, Mayerl C, Wimpissinger TF, Feichtinger J, Zelger B, et al. The immunology of fibrosis. Annu Rev Immunol 2013;31:107-35 doi 10.1146/annurevimmunol-032712-095937.

30. Ueha S, Shand FH, Matsushima K. Cellular and molecular mechanisms of chronic inflammation-associated organ fibrosis. Front Immunol 2012;3:71 doi 10.3389/fimmu.2012.00071.

31. Sun X, Glynn DJ, Hodson LJ, Huo C, Britt K, Thompson EW, et al. CCL2-driven inflammation increases mammary gland stromal density and cancer susceptibility in a transgenic mouse model. Breast Cancer Res 2017;19(1):4 doi 10.1186/s13058-0160796-z.

32. Qian BZ, Li J, Zhang H, Kitamura T, Zhang J, Campion LR, et al. CCL2 recruits inflammatory monocytes to facilitate breast-tumour metastasis. Nature 2011;475(7355):222-5 doi 10.1038/nature10138. 
876

877

878

879

880

881

882

883

884

885

886

887

888

889

890

891

892

893

894

895

896

897

898

899

900

901

902

903

904

905

906

907

908

909

910

911

912

913

914

915

916

917

918

919

920

921

922

923

924

925

926

33. Lohela M, Casbon AJ, Olow A, Bonham L, Branstetter D, Weng N, et al. Intravital imaging reveals distinct responses of depleting dynamic tumor-associated macrophage and dendritic cell subpopulations. Proc Natl Acad Sci U S A 2014;111(47):E5086-95 doi 10.1073/pnas. 1419899111.

34. Ruffell B, Chang-Strachan D, Chan V, Rosenbusch A, Ho CM, Pryer N, et al. Macrophage IL-10 blocks CD8+ T cell-dependent responses to chemotherapy by suppressing IL-12 expression in intratumoral dendritic cells. Cancer Cell 2014;26(5):62337 doi 10.1016/j.ccell.2014.09.006.

35. DeNardo DG, Brennan DJ, Rexhepaj E, Ruffell B, Shiao SL, Madden SF, et al. Leukocyte complexity predicts breast cancer survival and functionally regulates response to chemotherapy. Cancer Discov 2011;1(1):54-67 doi 10.1158/2159-8274.CD10-0028.

36. Nemkov T, D'Alessandro A, Hansen KC. Three-minute method for amino acid analysis by UHPLC and high-resolution quadrupole orbitrap mass spectrometry. Amino acids 2015;47(11):2345-57.

37. Oxlund H, Barckman M, Ørtoft G, Andreassen T. Reduced concentrations of collagen cross-links are associated with reduced strength of bone. Bone 1995;17(4):S365-S71.

38. Gruosso T, Gigoux M, Manem VSK, Bertos N, Zuo D, Perlitch I, et al. Spatially distinct tumor immune microenvironments stratify triple-negative breast cancers. J Clin Invest 2019;129(4):1785-800 doi 10.1172/JCI96313.

39. Finak G, Bertos N, Pepin F, Sadekova S, Souleimanova M, Zhao H, et al. Stromal gene expression predicts clinical outcome in breast cancer. Nat Med 2008;14(5):518-27 doi 10.1038/nm1764.

40. Salvador F, Martin A, Lopez-Menendez C, Moreno-Bueno G, Santos V, VazquezNaharro A, et al. Lysyl oxidase-like protein LOXL2 promotes lung metastasis of breast cancer. Cancer Res 2017 doi 10.1158/0008-5472.CAN-16-3152.

41. Eyre DR, Paz MA, Gallop PM. Cross-linking in collagen and elastin. Annual review of biochemistry 1984;53(1):717-48.

42. Lin EY, Nguyen AV, Russell RG, Pollard JW. Colony-stimulating factor 1 promotes progression of mammary tumors to malignancy. J Exp Med 2001;193(6):727-39 doi DOI 10.1084/jem.193.6.727.

43. Strachan DC, Ruffell B, Oei Y, Bissell MJ, Coussens LM, Pryer N, et al. CSF1R inhibition delays cervical and mammary tumor growth in murine models by attenuating the turnover of tumor-associated macrophages and enhancing infiltration by CD8(+) T cells. Oncoimmunology 2013;2(12):e26968 doi 10.4161/onci.26968.

44. Lin EY, Jones JG, Li P, Zhu L, Whitney KD, Muller WJ, et al. Progression to malignancy in the polyoma middle T oncoprotein mouse breast cancer model provides a reliable model for human diseases. Am J Pathol 2003;163(5):2113-26 doi 10.1016/S00029440(10)63568-7.

45. Takaluoma K, Lantto J, Myllyharju J. Lysyl hydroxylase 2 is a specific telopeptide hydroxylase, while all three isoenzymes hydroxylate collagenous sequences. Matrix biology 2007;26(5):396-403.

46. Yau C, Esserman L, Moore DH, Waldman F, Sninsky J, Benz CC. A multigene predictor of metastatic outcome in early stage hormone receptor-negative and triple-negative breast cancer. Breast Cancer Res 2010;12(5):R85 doi 10.1186/bcr2753.

47. Gyorffy B, Lanczky A, Eklund AC, Denkert C, Budczies J, Li Q, et al. An online survival analysis tool to rapidly assess the effect of 22,277 genes on breast cancer prognosis using microarray data of 1,809 patients. Breast Cancer Res Treat 2010;123(3):725-31 doi 10.1007/s10549-009-0674-9.

48. Noy R, Pollard JW. Tumor-associated macrophages: from mechanisms to therapy. Immunity 2014;41(1):49-61 doi 10.1016/j.immuni.2014.06.010. 
927

928

929

930

931

932

933

934

935

936

937

938

939

940

941

942

943

944

945

946

947

948

949

950

951

952

953

954

955

956

957

958

959

960

961

962

963

964

965

966

967

968

969

970

971

972

973

49. Lin EY, Li JF, Gnatovskiy L, Deng Y, Zhu L, Grzesik DA, et al. Macrophages regulate the angiogenic switch in a mouse model of breast cancer. Cancer Res 2006;66(23):1123846 doi 10.1158/0008-5472.CAN-06-1278.

50. Gilkes DM, Bajpai S, Chaturvedi P, Wirtz D, Semenza GL. Hypoxia-inducible factor 1 (HIF-1) promotes extracellular matrix remodeling under hypoxic conditions by inducing P4HA1, P4HA2, and PLOD2 expression in fibroblasts. J Biol Chem 2013;288(15):1081929 doi $10.1074 /$ jbc.M112.442939.

51. Wong CC, Gilkes DM, Zhang H, Chen J, Wei H, Chaturvedi P, et al. Hypoxia-inducible factor 1 is a master regulator of breast cancer metastatic niche formation. Proc Natl Acad Sci U S A 2011;108(39):16369-74 doi 10.1073/pnas.1113483108.

52. van der Slot AJ, Zuurmond A-M, Bardoel AF, Wijmenga C, Pruijs HE, Sillence DO, et al. Identification of PLOD2 as telopeptide lysyl hydroxylase, an important enzyme in fibrosis. Journal of Biological Chemistry 2003;278(42):40967-72.

53. Lu P, Takai K, Weaver VM, Werb Z. Extracellular matrix degradation and remodeling in development and disease. Cold Spring Harb Perspect Biol 2011;3(12) doi 10.1101/cshperspect.a005058.

54. Ahn Y, Sanderson BW, Klein OD, Krumlauf R. Inhibition of Wnt signaling by Wise (Sostdc1) and negative feedback from Shh controls tooth number and patterning. Development 2010;137(19):3221-31 doi 10.1242/dev.054668.

55. Hsiao EC, Boudignon BM, Chang WC, Bencsik M, Peng J, Nguyen TD, et al. Osteoblast expression of an engineered Gs-coupled receptor dramatically increases bone mass. Proc Natl Acad Sci U S A 2008;105(4):1209-14 doi 10.1073/pnas.0707457105.

56. Guy CT, Cardiff RD, Muller WJ. Induction of mammary tumors by expression of polyomavirus middle T oncogene: a transgenic mouse model for metastatic disease. Mol Cell Biol 1992;12(3):954-61.

57. Avery NC, Sims TJ, Bailey AJ. Quantitative determination of collagen cross-links. Extracellular Matrix Protocols: Second Edition 2009:103-21.

58. Clasquin MF, Melamud E, Rabinowitz JD. LC-MS Data Processing with MAVEN: A Metabolomic Analysis and Visualization Engine. Current Protocols in Bioinformatics 2012:14.1. 1-.1. 23.

59. Cerami E, Gao J, Dogrusoz U, Gross BE, Sumer SO, Aksoy BA, et al. The cBio cancer genomics portal: an open platform for exploring multidimensional cancer genomics data. Cancer Discov 2012;2(5):401-4 doi 10.1158/2159-8290.CD-12-0095.

60. Gao J, Aksoy BA, Dogrusoz U, Dresdner G, Gross B, Sumer SO, et al. Integrative analysis of complex cancer genomics and clinical profiles using the cBioPortal. Sci Signal 2013;6(269):pl1 doi 10.1126/scisignal.2004088.

61. Curtis C, Shah SP, Chin SF, Turashvili G, Rueda OM, Dunning MJ, et al. The genomic and transcriptomic architecture of 2,000 breast tumours reveals novel subgroups. Nature 2012;486(7403):346-52 doi 10.1038/nature10983.

62. Berglund G, Elmstahl S, Janzon L, Larsson SA. The Malmo Diet and Cancer Study. Design and feasibility. J Intern Med 1993;233(1):45-51.

63. Manjer J, Carlsson S, Elmstahl S, Gullberg B, Janzon L, Lindstrom M, et al. The Malmo Diet and Cancer Study: representativity, cancer incidence and mortality in participants and non-participants. Eur J Cancer Prev 2001;10(6):489-99.

64. Cohen DA, Dabbs DJ, Cooper KL, Amin M, Jones TE, Jones MW, et al. Interobserver agreement among pathologists for semiquantitative hormone receptor scoring in breast carcinoma. Am J Clin Pathol 2012;138(6):796-802 doi 10.1309/AJCP6DKRND5CKVDD. 
Fig 1

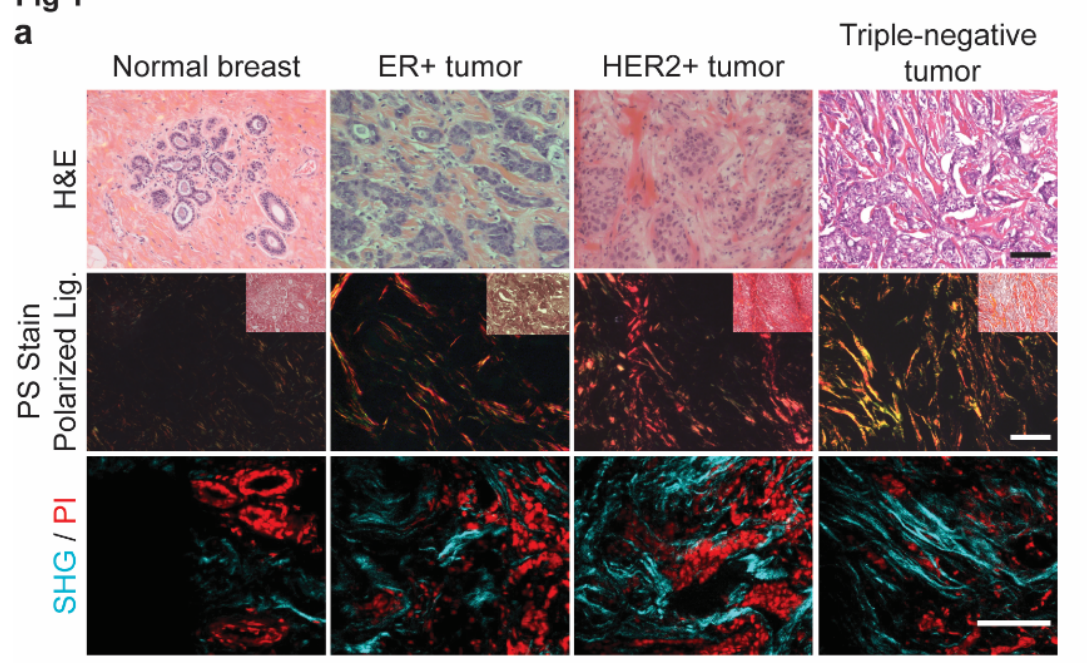

b

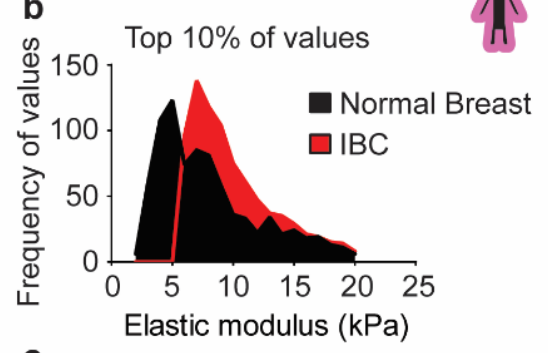

C
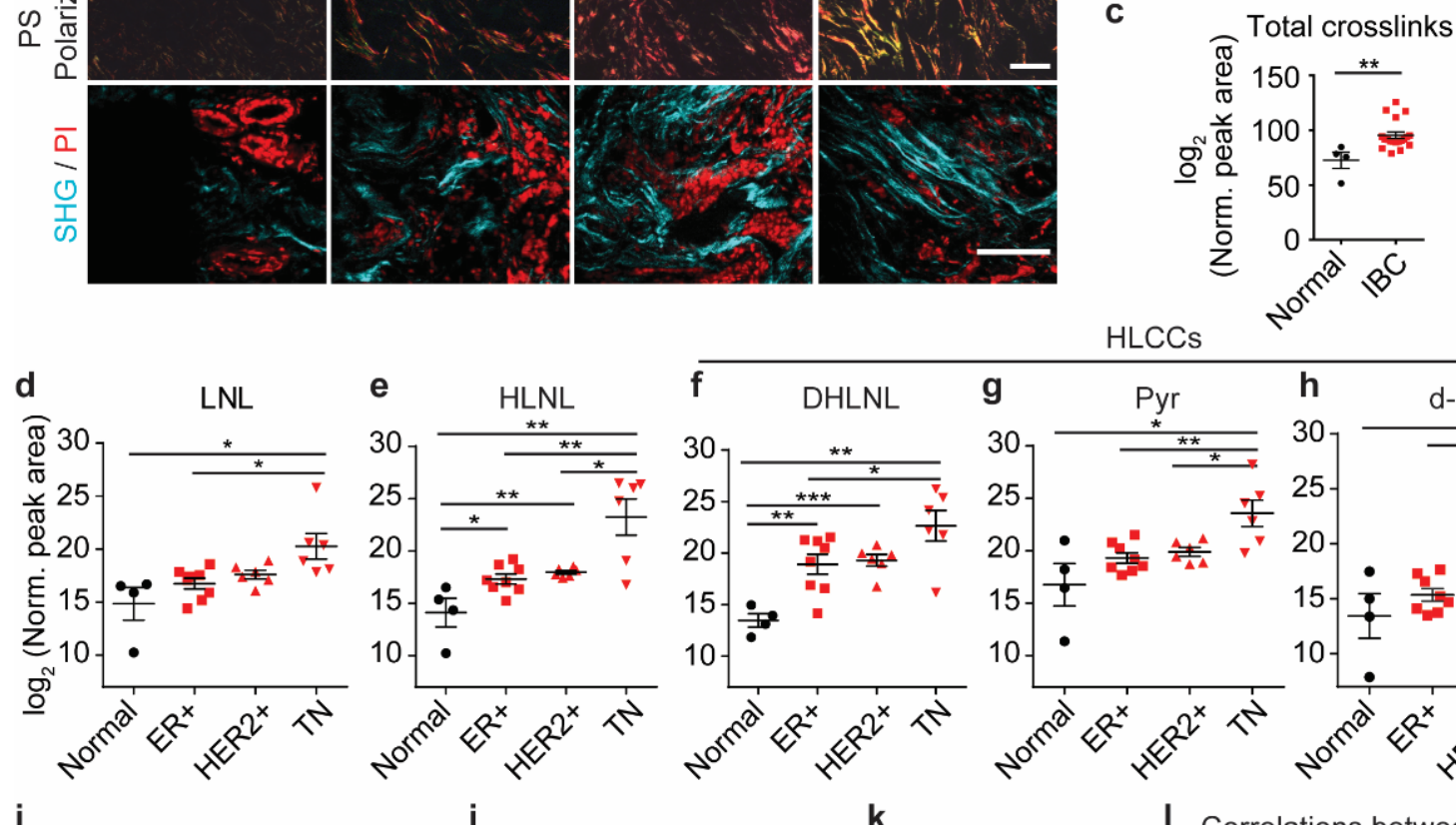

HLCCS
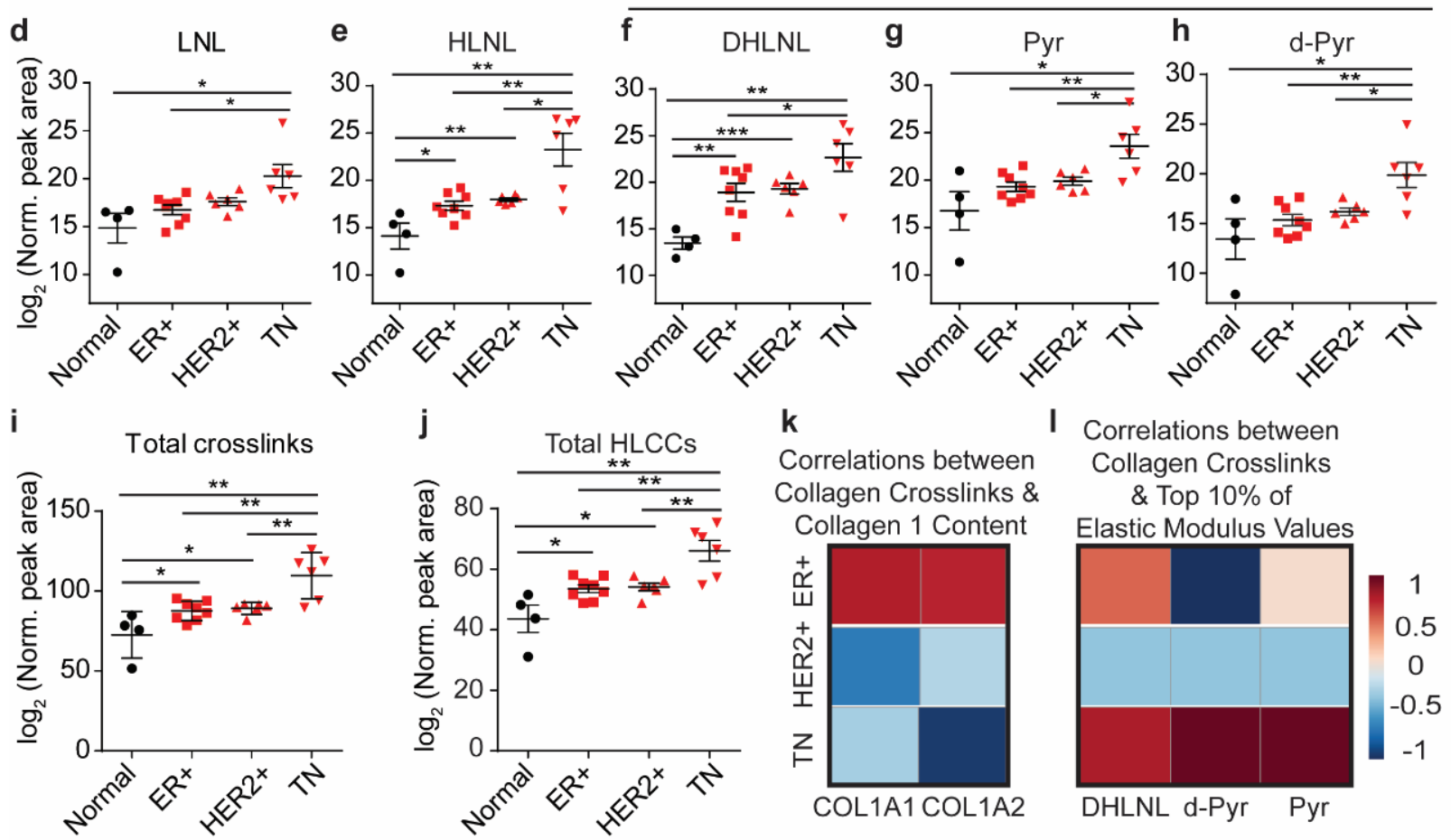

k

Correlations between

Collagen Crosslinks \&

Correlations between

Collagen Crosslinks

Collagen 1 Content

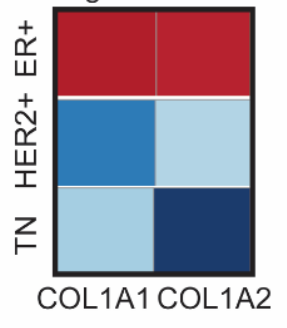

\& Top $10 \%$ of

Elastic Modulus Values

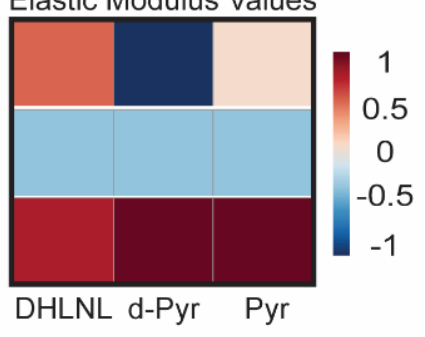

Figure 1: Hydroxylysine collagen crosslink abundance correlates with human breast cancer aggression (a) Representative images of normal breast tissue (normal breast; $n=4$ ) and invasive tumors diagnosed as estrogen receptor positive (ER+ tumor; $n=8)$, epidermal growth factor receptor two positive (HER2+ tumor; $n=6$ ) and triple negative (TN tumor; $n=6$ ). (Top row) Brightfield images of human breast tissue stained with hematoxylin and eosin (H\&E). (Middle row) Polarized light images of picrosirius red (PS) stained human breast tissue with an inset brightfield image shows relative levels of fibrillar collagen. (Bottom row) Two photon second harmonic generation (SHG) images of human breast tissue revealing collagen organization 
(turquoise) and propidium iodide (PI; red) stained nuclei. Scale bar, $100 \mu \mathrm{m}$. (b) The distribution of the top $10 \%$ of elastic modulus values of normal breast tissue $(n=10)$ and invasive breast carcinoma (IBC; $n=10$ ) measured by AFM microindentation. Statistical analysis was performing using Mann-Whitney $U$ test $\left({ }^{* * * *} p<0.0001\right)$. (c) Quantification of the abundance of all detected collagen crosslinks in normal breast tissue $(n=4)$ and in IBC tissues $(n=19)$ plotted as a scatter plot of individual samples with mean \pm SEM. Statistical analysis was performed using MannWhitney $U$ test $\left({ }^{* *} p<0.01\right)$. (d-h) Scatter plots showing individual and mean values \pm SEM of the levels of each LCC and HLCC crosslink measured in normal breast tissues, and in ER+, HER2+ and TN breast tumors. The total abundance of crosslinks (i) was calculated by summing all individual crosslinks and the total tissue HLCC abundance $(j)$ was calculated by summing DHLNL, Pyr, and d-Pyr and plotted as individual and mean values \pm SEM. All crosslink values are normalized to total collagen content (i.e. hydroxyproline abundance) and wet tissue weight and are plotted as $\log _{2}$ transformed normalized peak areas from LC-MS data. Statistical analysis of crosslinks was performed using one-way ANOVA test for overall analysis and unpaired t-test was used for individual comparisons $\left({ }^{*} p<0.05\right.$; $\left.{ }^{* *} p<0.01\right)$. $(\mathbf{k}, \mathbf{l})$ Heat maps of Spearman correlation coefficients indicating correlations between levels of total collagen crosslinks and collagen I content (k) and between levels of each HLCC and the top $10 \%$ of elastic modulus measurements (I) stratified by tumor subtype. 
Fig 2
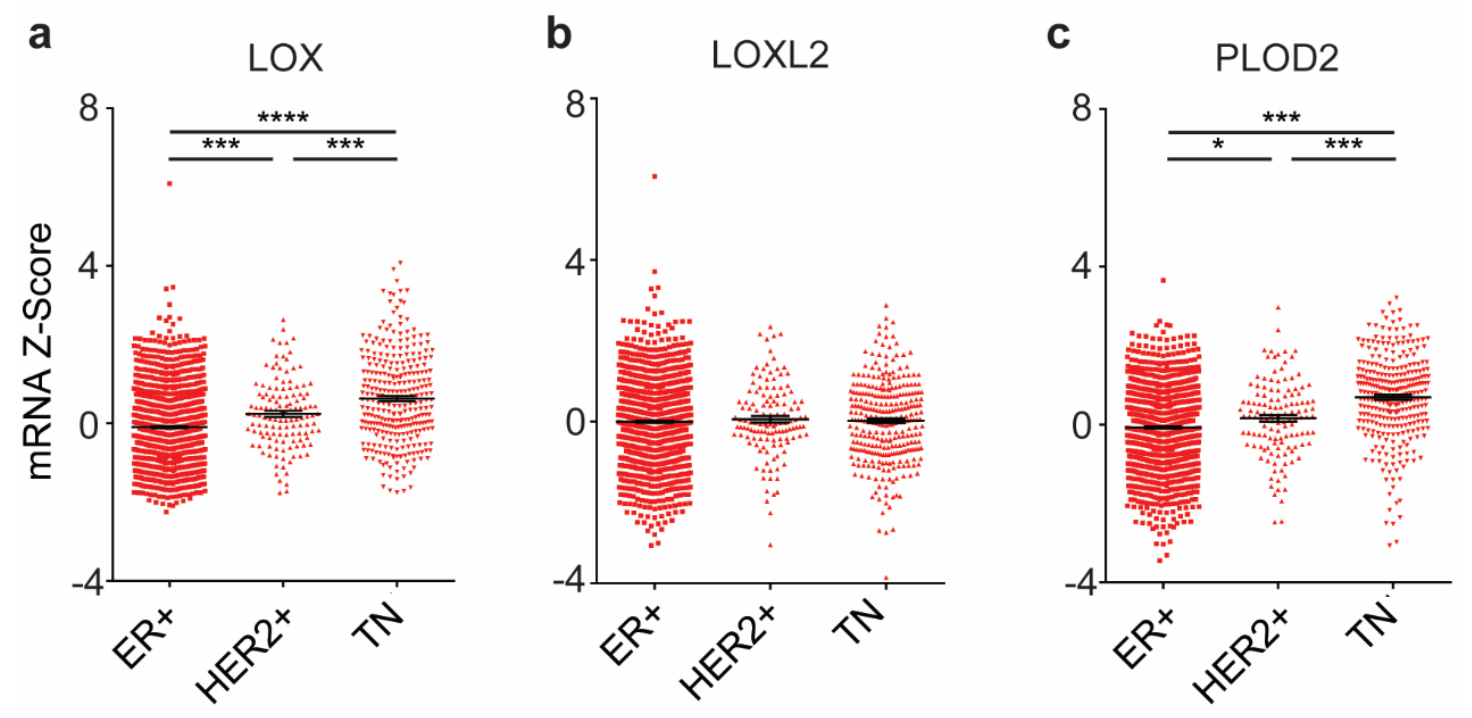

d
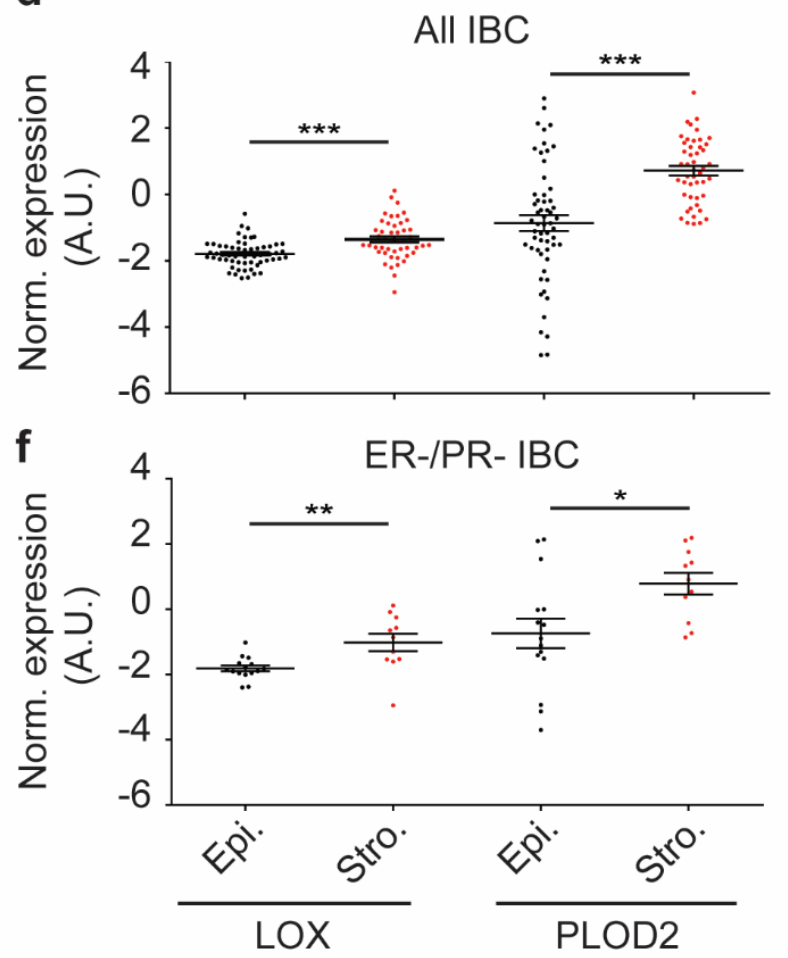

e

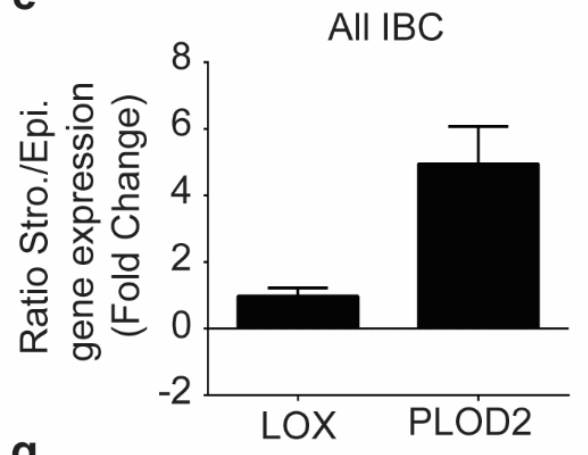

g

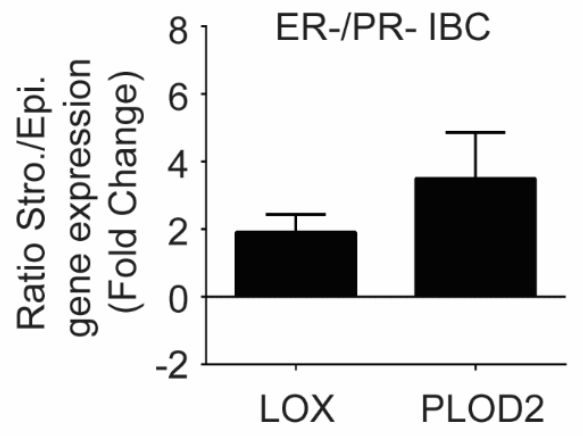


Figure 2: LOX and PLOD2 are enriched in TNBC and predominantly expressed by stromal cells. (a-c) Gene expression analysis of LOX (a), LOXL2 (b), and PLOD2 (c) stratified by $\mathrm{ER}^{+}(\mathrm{n}$ $=1355), \mathrm{HER}^{+}(n=127)$, and triple negative $(T N ; n=299)$ subtypes. Gene expression is plotted as a scatter plot of mRNA $z$ scores with the mean \pm SEM. Statistical analysis was performed using one-way ANOVA for overall analysis and unpaired t-test was used for individual comparisons ( ${ }^{*} p$ $\left.<0.05,{ }^{* *} p<0.01,{ }^{* * *} p<0.001,{ }^{* * * *} p<0.0001\right)$. (d) Scatter plot of individual and mean values \pm SEM comparing LOX $(n=47)$ and PLOD2 $(n=57)$ gene expression in microdissected epithelial and stromal compartments of human invasive breast carcinomas. Statistical analysis was performed using Mann-Whitney $U$ test $\left({ }^{* * *} p<0.0001\right)$. (e) Quantification of LOX and PLOD2 gene expression fold change from (d) in stromal cells relative to epithelial cells. (f-g) Restriction of the stromal/epithelial gene expression analysis in (d) and (e) to estrogen receptor (ER) negative and progesterone receptor (PR) negative samples. (LOX $n=11$, PLOD2 $n=15$ ). Statistical analysis was performed using Mann-Whitney $U$ test $\left({ }^{*} p<0.05,{ }^{* *} p<0.01\right)$. 
Fig 3

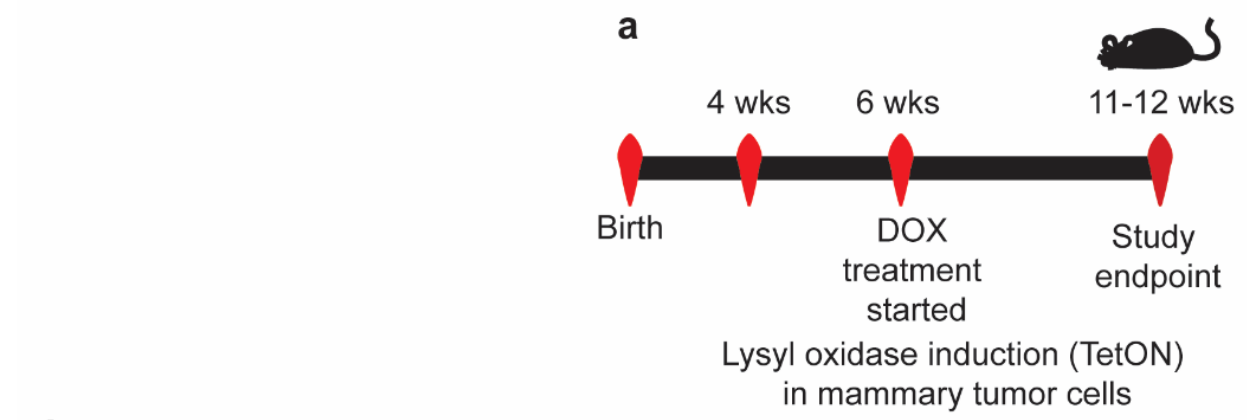

b

in mammary tumor cells
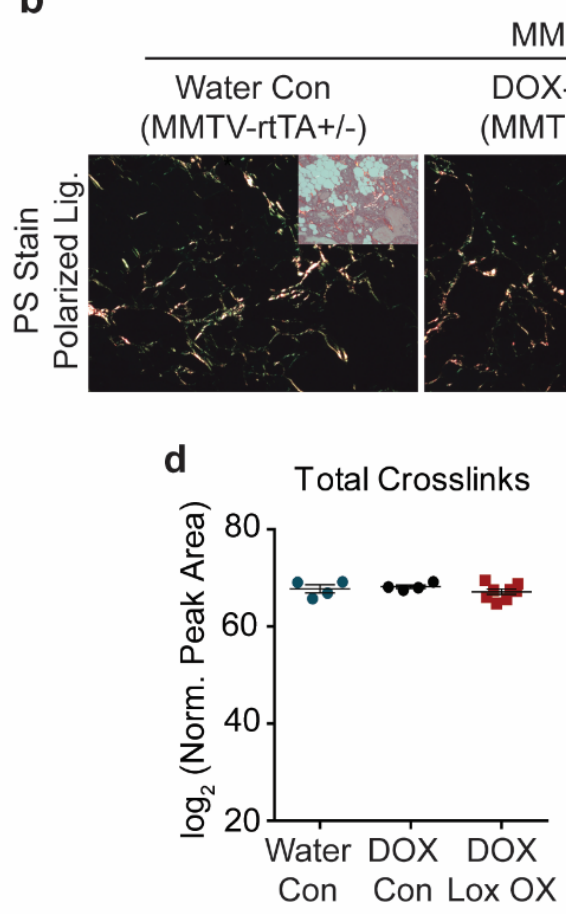

MMTV-PyMT

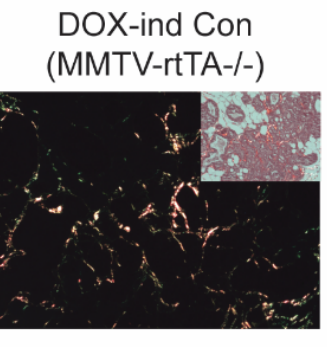

e

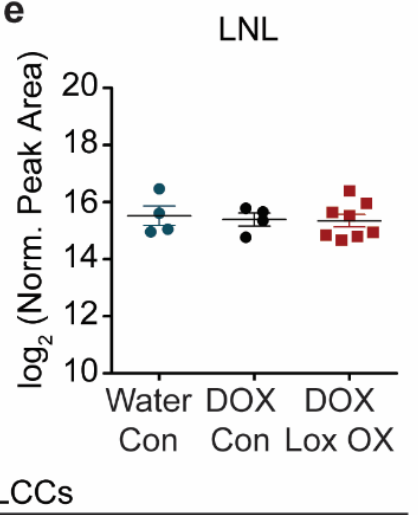

Total HLCCs
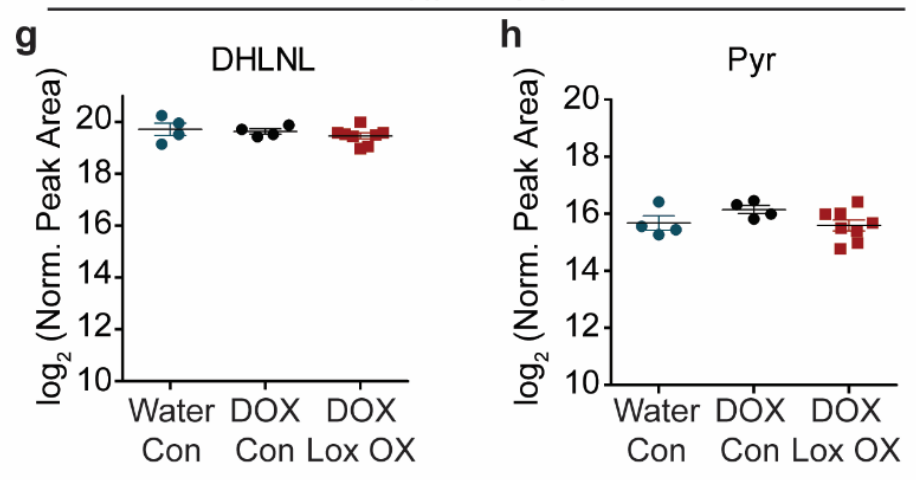

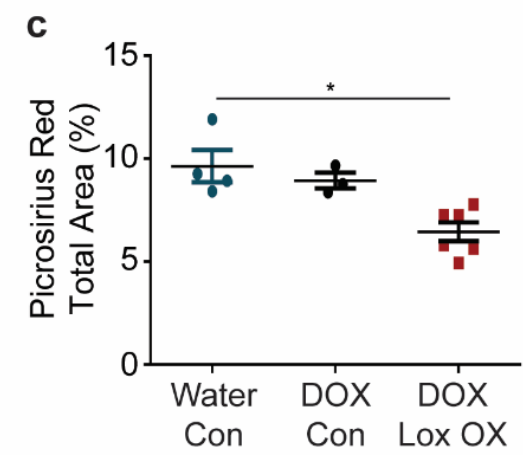

f
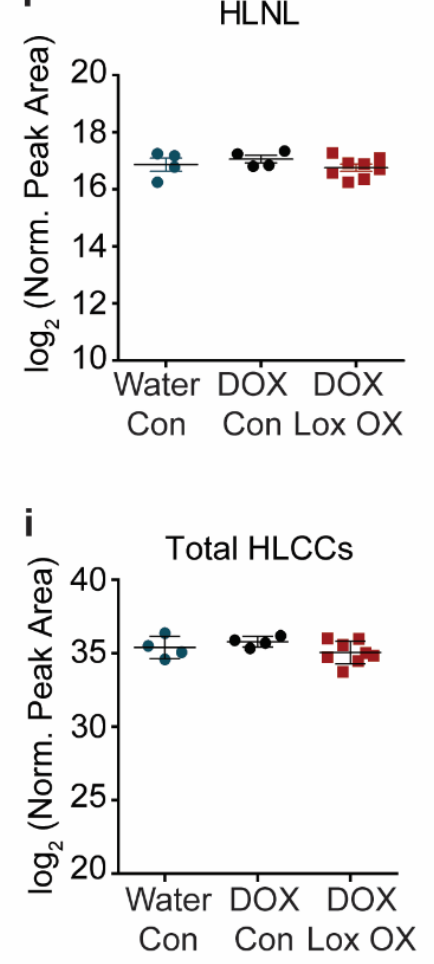
Figure 3: Epithelial-derived collagen crosslinking enzymes fail to induce collagen crosslinking. (a) Schematic depicting the experimental strategy used to induce epithelial Lox overexpression. (b) Polarized light images with brightfield inset of picrosirius red stained murine mammary tissues. (c) Quantification of percent area of picrosirius red staining per field of view, plotted as a scatter plot of the mean for each animal \pm SEM. Statistical analysis was performed using Kruskal-Wallis one-way ANOVA ( ${ }^{*} p<0.05$ ). (d) Scatter plot showing individual and mean values \pm SEM of total tissue collagen crosslinks in PyMT controls (Water $n=4$, DOX $n=4$ ) and PyMT epithelial Lox overexpression $(n=8)$. (e-h) Scatter plots showing individual and mean values \pm SEM for each LCC and HLCC collagen crosslink measured in PyMT control and PyMT epithelial Lox overexpression tumor tissue. (i) Scatter plot showing individual and mean values \pm SEM of total HLCCs calculated as the sum of DHLNL and Pyr crosslinks. Quantity of crosslinks per tissue was calculated normalizing crosslinks to total collagen content (i.e., hydroxyproline abundance) and wet tissue weight. Values were plotted as $\log _{2}$ transformed normalized peak areas as quantified from LC-MS data. Statistical analyses for crosslinking data were performed using one-way ANOVA for overall comparison and unpaired t-test for individual comparisons $\left({ }^{*} p\right.$ $\left.<0.05,{ }^{* *} p<0.01,{ }^{* * *} p<0.001,{ }^{* * * *} p<0.0001\right)$. 
Fig 4

a
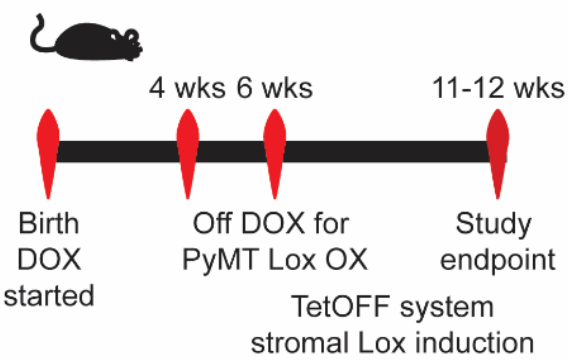

C

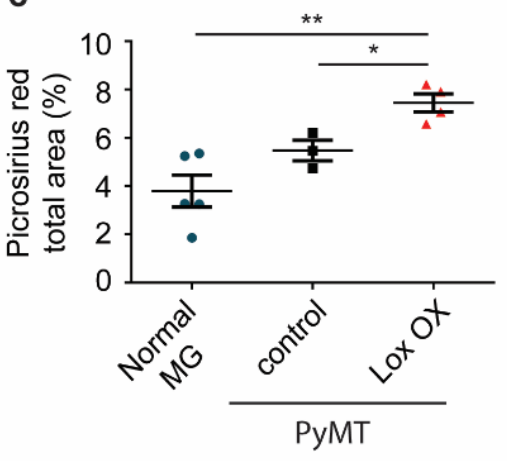

b

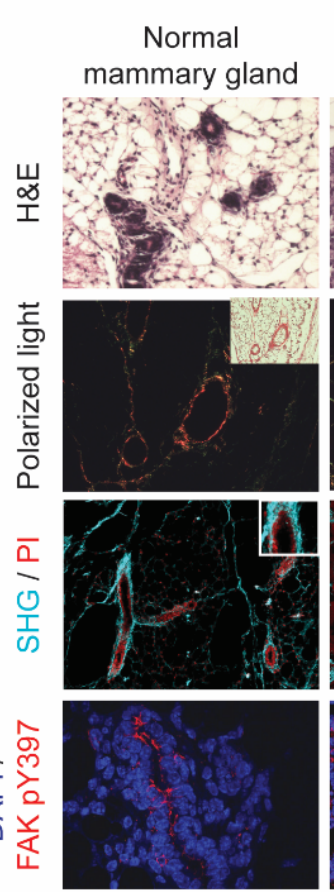

e d

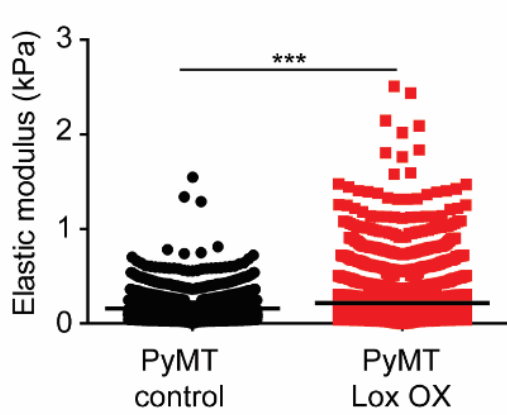

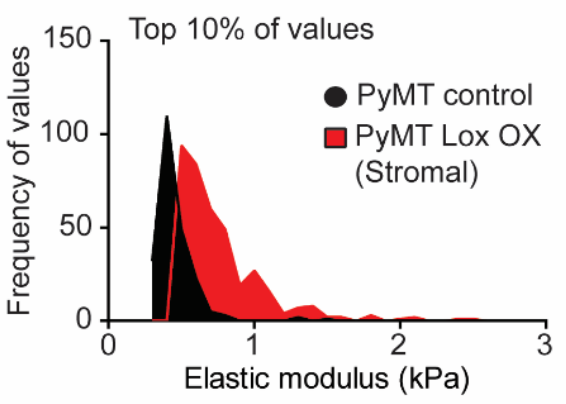
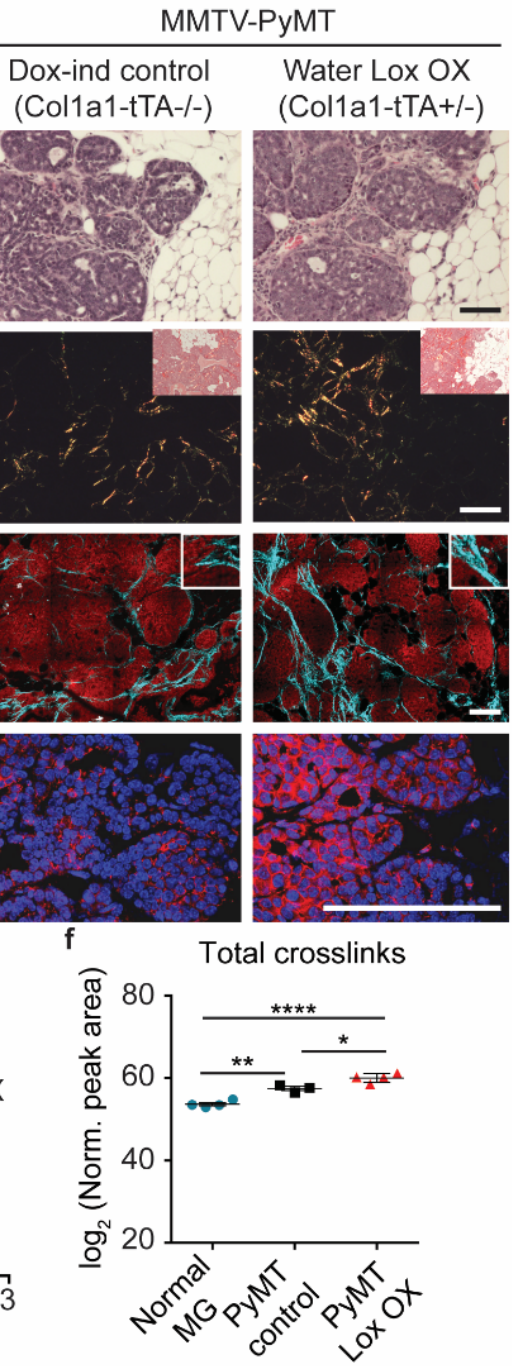

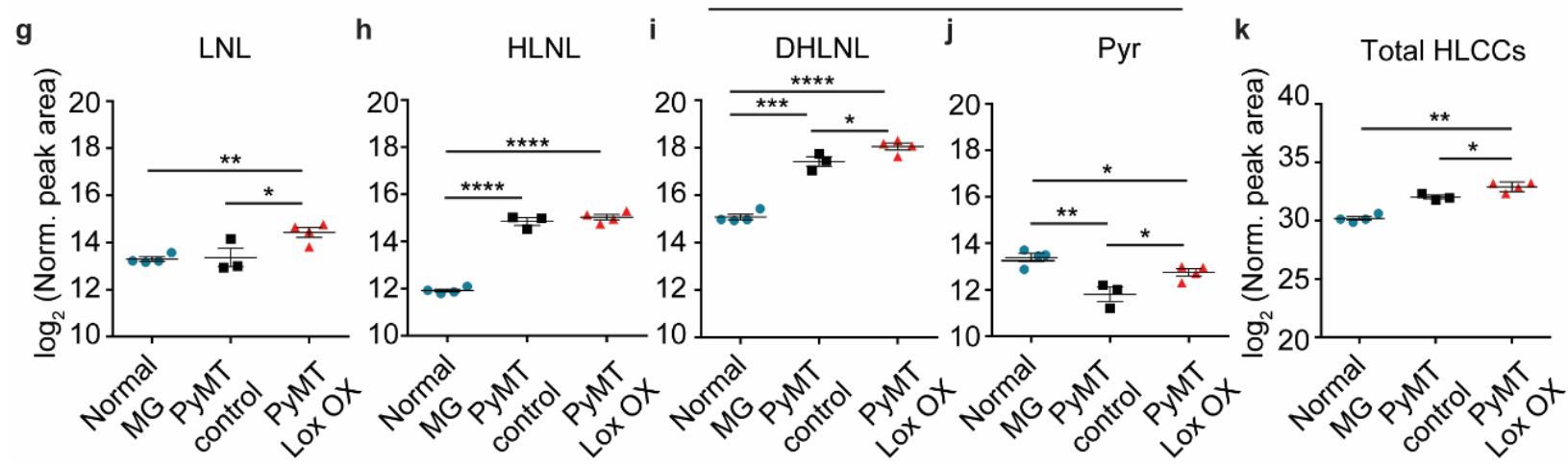


Figure 4: Stromal-derived LOX regulates collagen crosslinking and stiffening. (a) Schematic depicting the experimental strategy used to induce stromal Lox overexpression. (b) Representative images of normal murine mammary gland and PyMT control and stromal Lox overexpressing tumor tissues. (Top row) Brightfield images of H\&E stained murine mammary tissues. (Second row) Polarized light images with brightfield inset of picrosirius red stained murine mammary tissues. (Third row) Two photon second harmonic generation (SHG) images of murine mammary tissues revealing collagen organization (turquoise) and propidium iodide (PI; red) stained nuclei. (Bottom row) Confocal images of mammary tissue stained with anti-FAK pY397 monoclonal antibody (red) and DAPI (blue; nuclei). Scale bars are all $100 \mu \mathrm{m}$. (c) Quantification of fibrillar collagen by picrosirius red staining by percent area per field of view. The mean of 3-5 regions was calculated and plotted for each animal \pm SEM. Statistical analysis was performed using Kruskall-Wallis one-way ANOVA ( $\left.{ }^{*} p<0.05\right)$ for overall relationship and unpaired t-test for comparing individual groups $\left({ }^{*} p<0.05,{ }^{* *} p<0.005\right)$. (d) Scatter plot showing individual values and mean of the top $10 \%$ of elastic modulus measurements performed by AFM microindentation on the mammary stroma from mice with PyMT-induced tumors (PyMT Control; $\mathrm{n}=3$ mice) as compared to the stroma in PyMT-induced tumors in which lysyl oxidase was elevated in stromal cells (PyMT Lox OX; $n=4$ mice). Values reflect measurements taken from 35 individual force map regions per mammary gland. Statistical analyses were performed using Mann-Whitney $U$ test $\left.{ }^{* * *} p<0.001\right)$. (e) Histogram showing the distribution of the top $10 \%$ of elastic modulus measurements by AFM microindentation in PyMT control and Lox OX tumors. Statistical analysis was performed using Mann-Whitney $U$ test $\left({ }^{* * *} p<0.0001\right)$. (f) Scatter plots showing individual and mean values \pm SEM of total collagen crosslink abundance in the normal murine mammary gland $(n=4)$ as compared to glands with doxycycline-induced PyMT tumors ( $n$ $=3$ mice per control group) and PyMT tumors in which stromal lysyl oxidase was elevated $(n=4$ mice per Lox OX group). (g-j) Scatter plots showing individual and mean values \pm SEM of LCC and HLCC crosslinks quantified in normal mammary gland, PyMT Control tumors and PyMT Lox OX tumors. (k) Scatter plot showing individual and mean values \pm SEM of total HLCCs calculated as the sum of DHLNL and Pyr crosslinks. Quantity of crosslinks per tissue was calculated normalizing crosslinks to total collagen content (i.e., hydroxyproline abundance) and wet tissue weight. Values were plotted as $\log _{2}$ transformed normalized peak areas as quantified from LCMS data. Statistical analyses for crosslinking data were performed using one-way ANOVA for overall comparison and unpaired t-test for individual comparisons $\left({ }^{*} p<0.05,{ }^{* *} p<0.01,{ }^{* * *} p<\right.$ $\left.0.001,{ }^{* * * *} p<0.0001\right)$. 
Fig 5
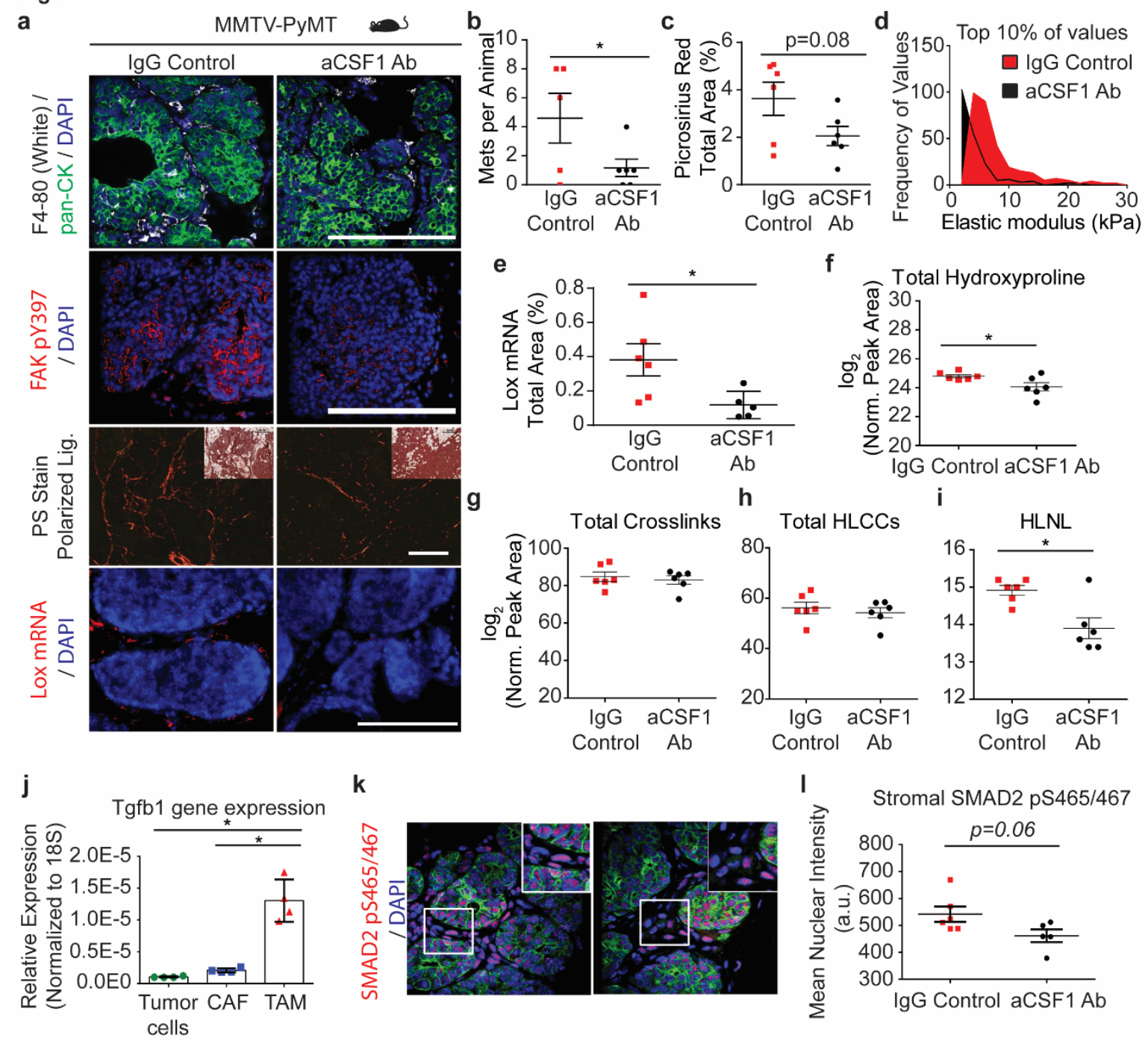

$$
\text { m }
$$

n
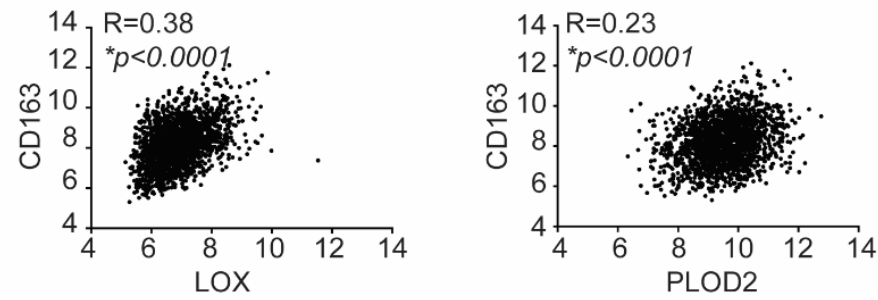

o

p

pSMAD2 Ser465/467 CD68
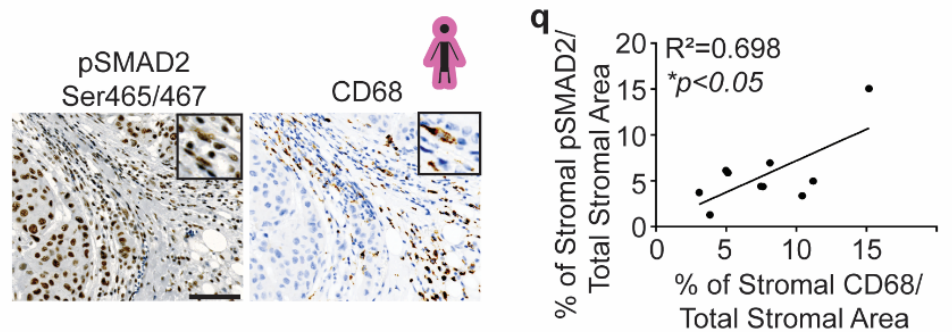

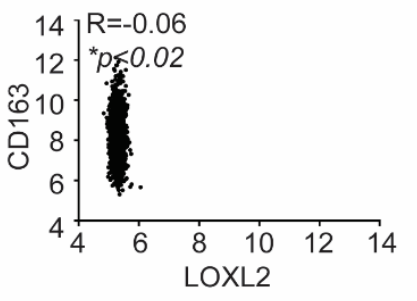

$\mathbf{r}$

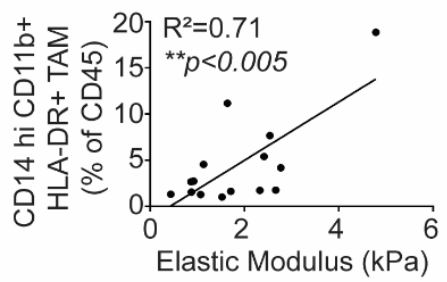


Figure 5: Tumor infiltrating macrophages secrete TGFb to activate stromal-mediated collagen crosslinking. (a) Representative images of PyMT tumor tissue from 8 weeks of age mice treated with anti-CSF1 blocking antibody or lgG1 control. (Top row) lgG1 treated $(n=6)$ and anti-CSF1 treated $(n=5)$ PyMT tumor tissue stained for pan-cytokeratin (green) marking epithelial cells, F4/80 (white) marking tumor infiltrating macrophages, and DAPI marking nuclei (blue). (Second row) IgG1 treated $(n=6)$ and anti-CSF1 treated $(n=5)$ PyMT tumor tissue stained for tyrosine 397 phosphorylated focal adhesion kinase (red) indicative of mechanosignaling and DAPI marking nuclei (blue). (Third row) Polarized light images with brightfield inset of IgG1 treated $(n=6)$ and anti-CSF1 treated $(n=6)$ PyMT tumor tissue stained with picrosirius red to visualize fibrillar collagen. (Bottom row) Lox mRNA in situ hybridization in IgG1 treated $(n=6)$ and anti-CSF1 treated $(n=5)$ PyMT tumor tissue and DAPI marking nuclei (blue). Scale bar for all images is $100 \mu \mathrm{m}$. (b) Quantification of the number of metastatic colonies in the lung tissues from lgG1 treated $(n=5)$ and anti-CSF1 treated $(n=6)$ mice at 11 weeks of age via PyMT IHC assessing 5 layers (5 micron section; 5 sections per layer; $50-100$ microns steps). Statistical analysis was performed using unpaired t-test $\left({ }^{*} p<0.05\right)$. (c) Quantification of fibrillar collagen by picrosirius red staining by percent area per field of view in 8 week old mice treated with anti-CSF1 blocking antibody or IgG1 control. The mean of 3-4 regions was calculated and plotted for each animal \pm SEM. Statistical analysis was performed using unpaired $t$-test $(p=$ 0.08). (d) Histogram showing the distribution of the top $10 \%$ of elastic modulus measurements by AFM microindentation in PyMT IgG1 treated $(n=6)$ and anti-CSF1 treated $(n=4)$ tumors. Statistical analysis was performed using Mann-Whitney $U$ test $\left({ }^{* * * *} p<0.0001\right)$. (e) Quantification of Lox mRNA signal by percent area of signal per field of view in 8 week old mice treated with anti-CSF1 blocking antibody $(n=5)$ or IgG1 control $(n=6)$. The mean of 5-6 regions was calculated and plotted for each animal \pm SEM. Statistical analysis was performed using an unpaired t-test $\left({ }^{*} p<0.05\right)$. ( $\left.f-i\right)$ Scatter plots showing individual and mean values $\pm S E M$ of the levels of total hydroxyproline (collagen content) (f), total collagen crosslinks (g), HLCCs (h), and HLNL crosslinks (i) in 8 week old IgG1 treated and anti-CSF1 treated PyMT tumors. Quantity of crosslinks per tissue was calculated normalizing crosslinks to wet tissue weight. Values were plotted as $\log _{2}$ transformed normalized peak areas as quantified from LC-MS data. Statistical analysis was performed using unpaired t-test ( $\left.{ }^{*} p<0.05\right)$. (j) Quantification of Tgfb1 gene expression by RT-qPCR in tumor cells, cancer-associated fibroblasts, and macrophages sorted out from PyMT tumors $(n=4)$. Gene expression was normalized to $18 \mathrm{~S}$. Statistical analysis was performed using Kruskal-Wallis one-way ANOVA for overall comparison and Mann-Whitney $U$ test for individual comparisons ( $\left.{ }^{*} p<0.05,{ }^{* * *} p<0.001\right)$. (k) Representative images of PyMT tumor tissue from mice treated with $\operatorname{lgG} 1(n=6)$ and anti-CSF1 $(n=5)$ stained for pan-cytokeratin (green) marking epithelial cells, SMAD2 pS465/467 (red), and DAPI marking nuclei (blue). (I) Scatter plot showing individual and mean values \pm SEM of the mean nuclear intensity of pSMAD $^{S 465 / 467}$ in stromal cells of IgG1 treated $(n=6)$ and anti-CSF1 treated $(n=5)$ PyMT mice. The mean for each animal was calculated from 4-7 regions within the tumor. Statistical analysis was performed using unpaired t-test $(p=0.06)$. $(m-0)$ Scatter plot depicting the Spearman correlation of CD163 gene expression with LOX (m), PLOD2 (n), and LOXL2 (o) in human breast tumors $(n=1904)$. (p) Representative IHC images of serial human breast tumor sections stained

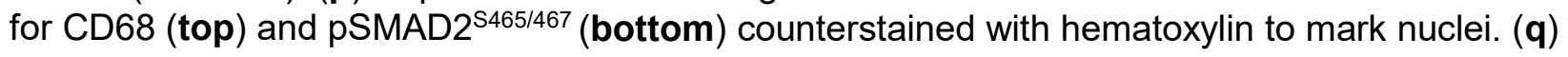
Scatter plot depicting the linear regression correlation of stromal pSMAD2 IHC staining with stromal CD68 IHC staining in human breast tumors $(n=10)$. (r) Scatter plot depicting the linear regression of $\mathrm{CD} 14^{+} \mathrm{CD} 11 \mathrm{~b}^{+} \mathrm{HLA}-\mathrm{DR}^{+}$tumor associated macrophage infiltrate with tumor elastic modulus as measured by AFM microindentation in human breast tumors $(n=15)$. 
bioRxiv preprint doi: https://doi.org/10.1101/2020.02.13.948141; this version posted February 13, 2020. The copyright holder for this preprint (which was not certified by peer review) is the author/funder, who has granted bioRxiv a license to display the preprint in perpetuity. It is made available under aCC-BY-NC-ND 4.0 International license.

Fig 6

a

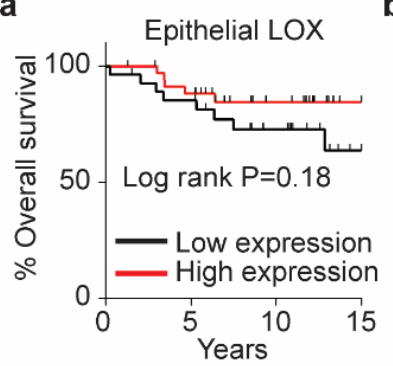

e
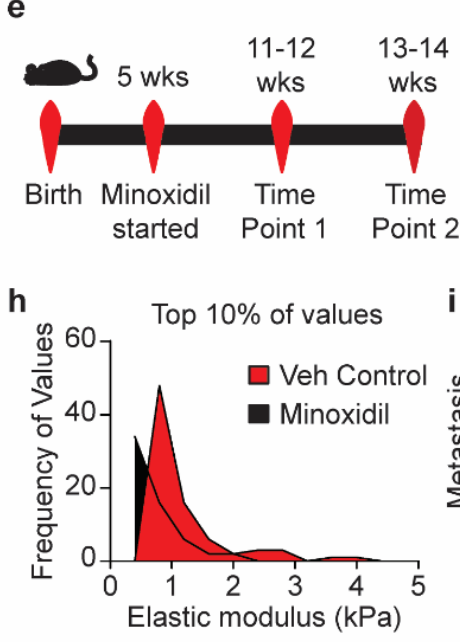

k

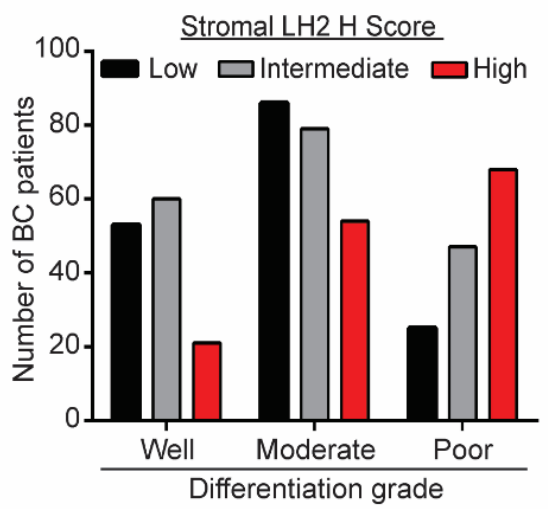

I

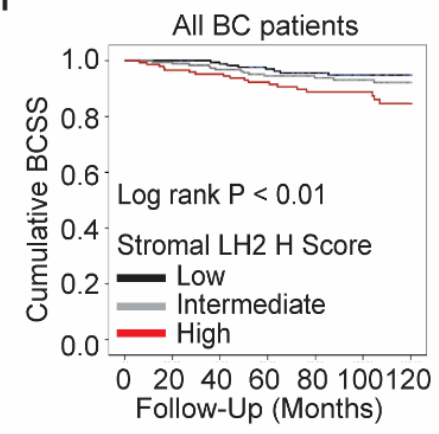

b

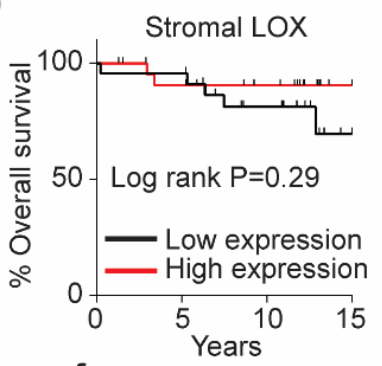

f

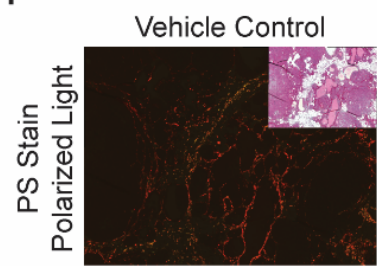

i
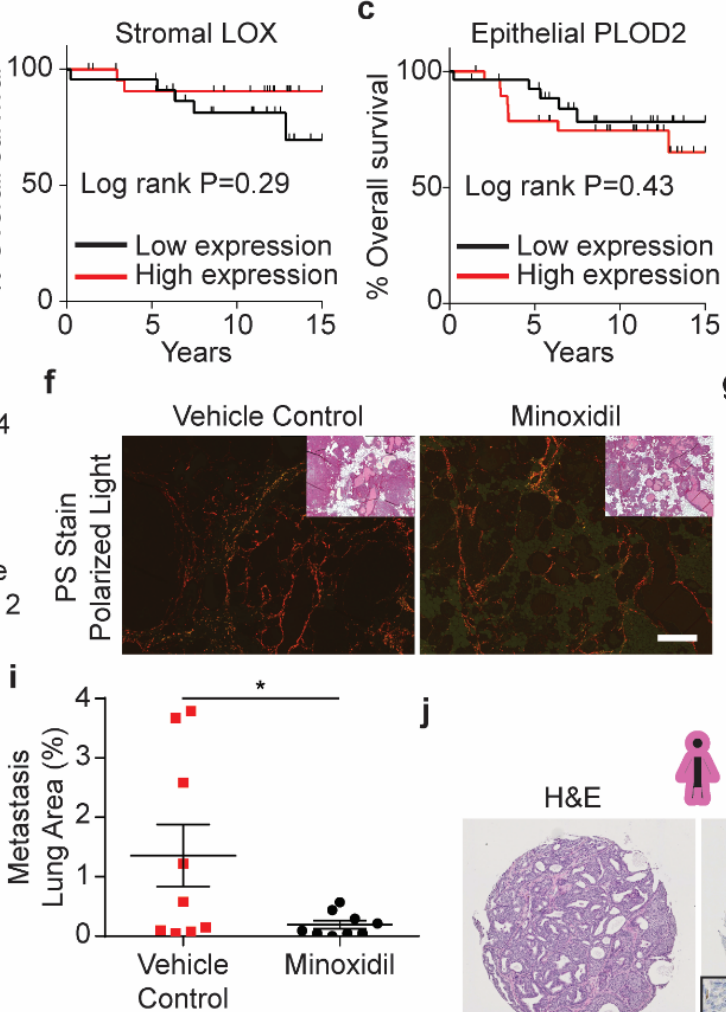

d

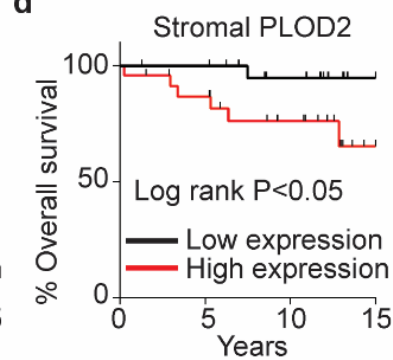

g
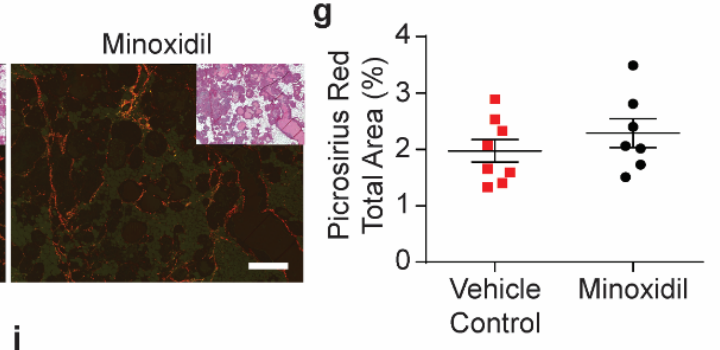

m

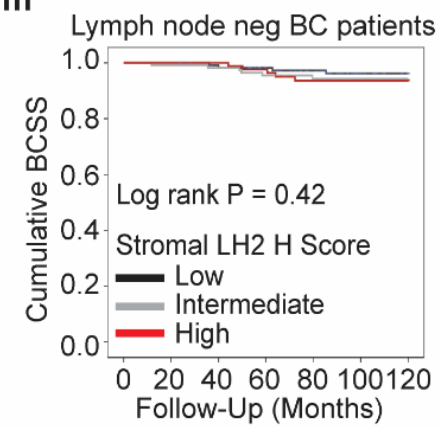

n

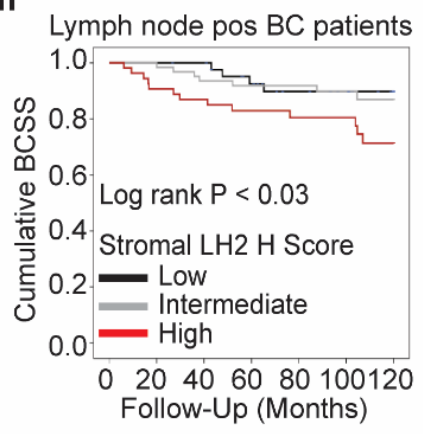


Figure 6: Stromal LH2 predicts poor patient outcomes. (a-b) Kaplan-Meier plots showing overall survival for patients based on levels of LOX expression in epithelial cells (low $n=28$, high $n=36$ ) (a) or stromal cells (low $n=23$, high $n=24$ ) (b). The median level of expression was defined as the cutoff for low and high expression. (c-d) Kaplan-Meier plots showing overall survival for patients based on levels PLOD2 expression in epithelial cells (low $n=28$, high $n=29$ ) (c) or stromal cells (low $n=23$, high $n=24$ ) $(d)$. The median level of expression was defined as the cutoff for low and high expression. (e) Schematic depicting the experimental timeline used to inhibit lysyl hydroxylase 2 in PyMT mice. (f) Representative polarized light images with brightfield insets of picrosirius red stained tumor tissue from PBS vehicle treated control $(n=8)$ and minoxidil treated $(n=7)$ PyMT mice. Scale bar is 100um. $(\mathbf{g})$ Quantification of fibrillar collagen by picrosirius red staining by percent area per field of view. The mean was calculated and plotted for each animal \pm SEM. (h) Histogram showing the distribution of the top $10 \%$ of elastic modulus measurements by AFM microindentation in PyMT control and Lox OX tumors. Statistical analysis was performed using Mann-Whitney $U$ test $\left({ }^{* * *} p<0.0001\right)$. (i) Scatter plot quantifying the area of lung sections occupied by metastases from vehicle treated $(n=9)$ and minoxidil treated $(n=8)$ mice at 13 weeks of age via H\&E staining and assessing 4 layers ( 5 micron section; 5 sections per layer; 50-100 microns steps). Statistical analysis was performed using a two-tailed unpaired t-test $\left({ }^{*} p<0.05\right)$. (j) Representative phase contrast images of sections from tissue microarrays (TMAs) of human breast cancers representing incident breast cancer cases collected and arrayed as 1-mm cores from each tumor. Sections were stained with Hematoxylin and Eosin (H\&E; top) and lysyl hydroxylase two (LH2; bottom) via immunohistochemistry. (k) Bar graphs showing clinical correlation between lysyl hydroxylase two (LH2) score as a function of tumor grade (see Table 1 for number of patients). LH2 IHC staining was assessed with the semi-quantitative stromal specific $\mathrm{H}$-score from $\mathrm{O}$ to 300 . The lowest tertile of $\mathrm{LH} 2 \mathrm{H}$-scores was defined as $\mathrm{H}$-scores between 0 and less or equal to 120 , the intermediate $\mathrm{H}$-score to above 120 and equal or less than 230, and the highest stromal LH2 score as above 230. For tumor grade and LH2 H score, statistical analysis was performed using a linear-by-linear association $\left({ }^{* * *} P<0.0001\right)$. (I) KaplanMeier curves indicating cumulative breast cancer specific survival (BCSS) based on stromal LH2 $\mathrm{H}$ score assessed in breast cancer patients up to 10 years after diagnosis (LH2 low $\mathrm{n}=175$, intermediate $n=188$, high $n=146)$. $(m)$ BCSS curves by stromal LH2 H score including only axillary lymph node negative patients (LH2 low $n=116$, intermediate $n=116$, high $n=90)$. (n) BCSS curves by stromal LH2 H score including only axillary lymph node positive patients (LH2 low $n=44$, intermediate $n=63$, high $n=54$ ). For Kaplan-Meier curves, statistical analyses were performed by LogRank test. 